Utah State University

DigitalCommons@USU

Mechanical and Aerospace Engineering Student Mechanical and Aerospace Engineering Student Publications and Presentations

$1-4-2021$

\title{
Practical Implementation of a General Numerical Lifting-Line Method
}

Cory D. Goates

Utah State University

Douglas F. Hunsaker

Utah State University, doug.hunsaker@usu.edu

Follow this and additional works at: https://digitalcommons.usu.edu/mae_stures

Part of the Mechanical Engineering Commons

\section{Recommended Citation}

Goates, C. D., and Hunsaker, D. F., "Practical Implementation of a General Numerical Lifting-Line Method," AIAA Scitech 2021Forum, AIAA, Virtual Event, 2021.

This Article is brought to you for free and open access by the Mechanical and Aerospace Engineering Student Research at DigitalCommons@USU. It has been accepted for inclusion in Mechanical and Aerospace Engineering Student Publications and Presentations by an authorized administrator of DigitalCommons@USU. For more information, please contact digitalcommons@usu.edu.

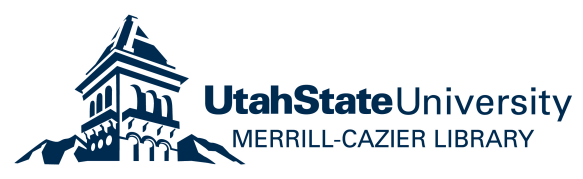




\title{
Practical Implementation of a General Numerical Lifting-Line Method
}

\author{
Cory D. Goates* and Douglas F. Hunsaker ${ }^{\dagger}$ \\ Utah State University, Logan, UT, 84321
}

\begin{abstract}
A general numerical lifting-line method provides corrections to overcome the singularities inherent in the lifting-line downwash integrals in certain cases. These singularities have previously limited the scope of lifting-line theory to straight wings not in sideslip; in all other cases, more traditional numerical approaches to solving Prandtl's hypothesis fail to grid converge. However, this general numerical lifting-line method grid converges even for swept wings and wings in sideslip. In the current work, we apply the general numerical lifting-line method to any number of wings with arbitrary geometry. We also provide a dimensional derivation of the basic general numerical lifting-line equations and discuss how airfoil section properties can be corrected for sweep. We develop a linearized system of equations and a nonlinear improvement method to solve the general numerical lifting-line equations. Results show that placing the lifting-line on the wing locus of aerodynamic centers, as done by others, may not yield the most accurate results. Comparisons with published data reveal that the general numerical lifting-line method can accurately predict the lift distribution for swept wings.
\end{abstract}

\section{Nomenclature}

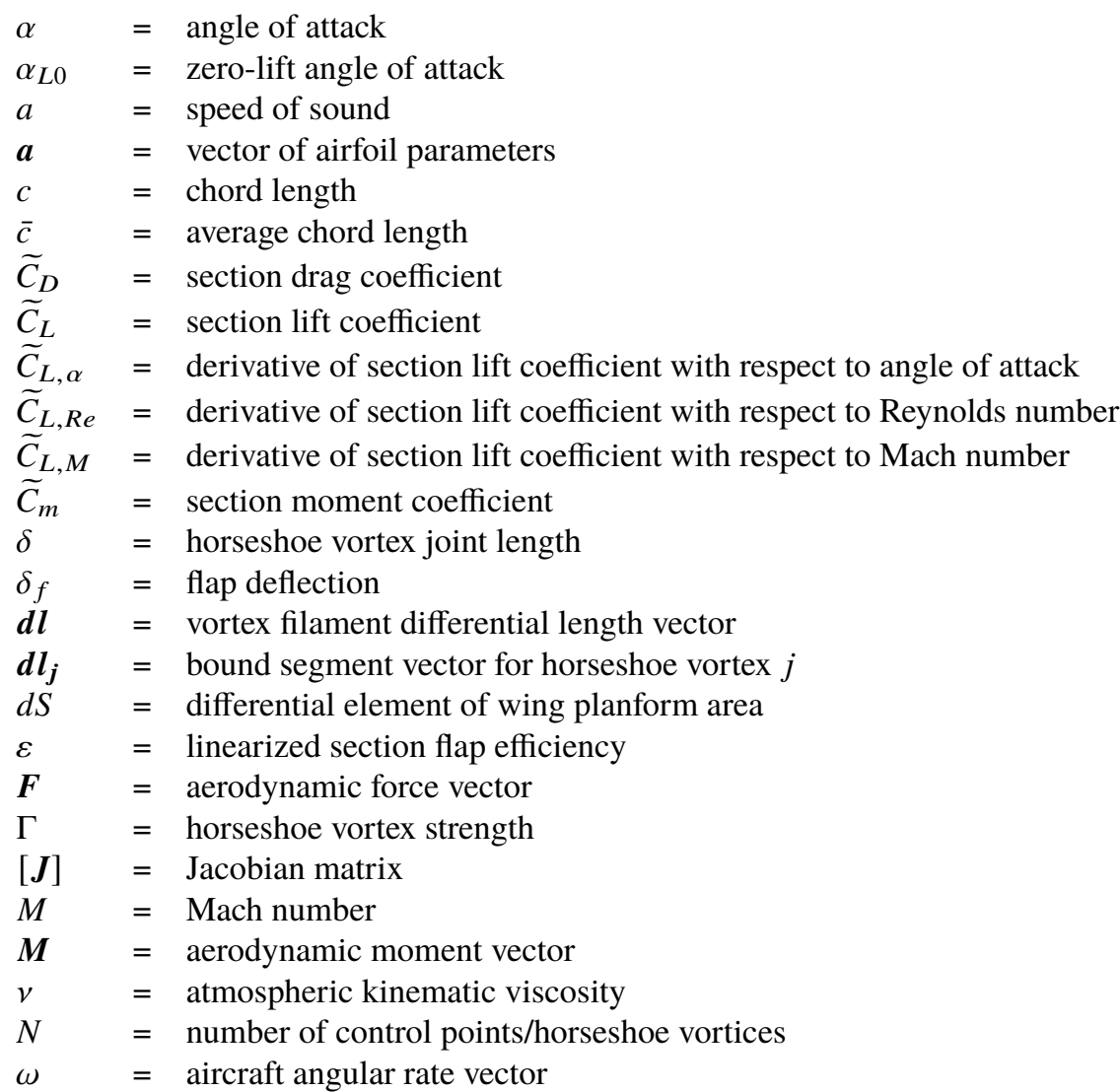

*PhD Candidate, Mechanical and Aerospace Engineering, 4130 Old Main Hill, AIAA Student Member.

${ }^{\dagger}$ Assistant Professor, Mechanical and Aerospace Engineering, 4130 Old Main Hill, AIAA Member. 


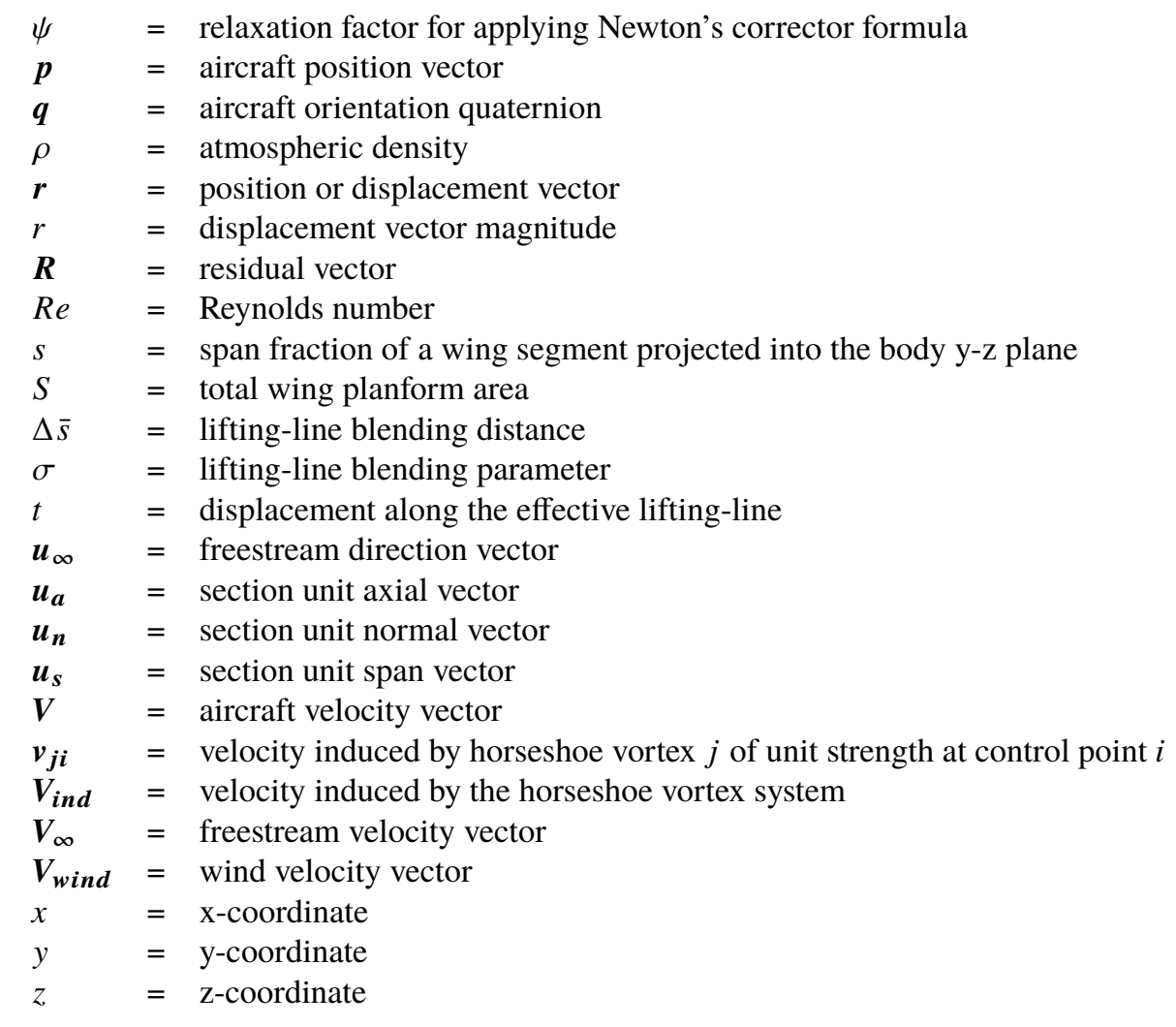

\section{Subscripts}

$b \quad=$ body frame

$i=$ control point index

$j=$ horseshoe vortex index

$L L=$ lifting-line

$\Lambda \quad=$ swept

$s \quad=\quad$ stability frame

$w=$ wind frame

$0=$ inbound horseshoe vortex node

$1=$ outbound horseshoe vortex node

Superscripts

' $=$ horseshoe vortex joint

$=$ effective lifting-line

Superscripts

$i=$ effective lifting-line index

\section{Introduction}

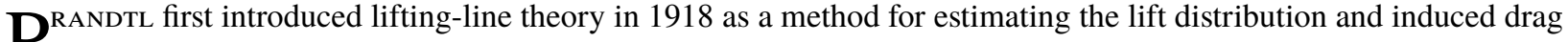
Pon a finite wing [1]. The central point of Prandtl's theory was modeling the wing as a variable-strength bound vortex segment, called the lifting-line. A vortex sheet lying in the plane of the wing then extended from the lifting-line into infinity in the direction of the chord. In order to satisfy Helmholtz's vortex theorems [2], the local strength of the trailing vortex sheet was defined as the negative of the change in strength of the bound vortex segment. This vortex sheet induces downwash on the bound vortex segment, making the local angle of attack a function of span. Prandtl hypothesized that the lift produced due to vortex circulation at any point along the wing must be the same as that produced by an infinite wing of the same cross section at the same local angle of attack. This is called the lifting-line hypothesis. Under this hypothesis, Prandtl's theory proves remarkably accurate for wings of aspect ratio greater than 4 at small angles of attack 
[3].

However, Prandtl only considered a single, straight wing with control points placed on the lifting-line [1]. Because of this, the velocity induced by the bound vortex on the control points is identically zero and so is left out. By including the velocity induced by the bound vortex, a wider range of geometries can be accounted for [4]. However, there are still two issues with Prandtl's classical formulation when wings with sweep or in sideslip are modeled. The first is that the integral for determining the velocity induced by the lifting-line at a point on the lifting-line contains a singularity which cannot be evaluated when the lifting-line has nonzero curvature at the point of interest [5]. Thus, non-straight wings cannot be modeled. The second issue is that the integral for determining the velocity induced by the vortex sheet at a point on the lifting-line contains a singularity which cannot be evaluated when the vortex sheet is not perpendicular to the lifting-line local to the point of interest [5, 6]. This means that, if the trailing vortex sheet is aligned with the freestream as is traditionally done [4], wings in sideslip or with sweep or dihedral cannot be modeled.

\section{A. Modern Numerical Lifting-Line Method}

In 2000, Phillips and Snyder presented a numerical analog to Prandtl's classical lifting-line theory, which we will refer to as the Phillips-Snyder (P-S) method [4]. Within the P-S method, Phillips and Snyder replace Prandtl's classical variable-strength bound vortex and trailing vortex sheet with a finite set of semi-infinite horseshoe vortices of constant strength, as depicted in Fig. [1 [4]. It is assumed in the P-S method that the lifting-line should be placed along the locus of aerodynamic centers (LAC) of the wing [3]. Each horseshoe vortex consists of a straight bound segment, lying approximately along a portion of the wing LAC, and two trailing segments extending behind the wing to infinity. A control point is also designated on each bound vortex segment at which the relevant equations are formulated and solved.

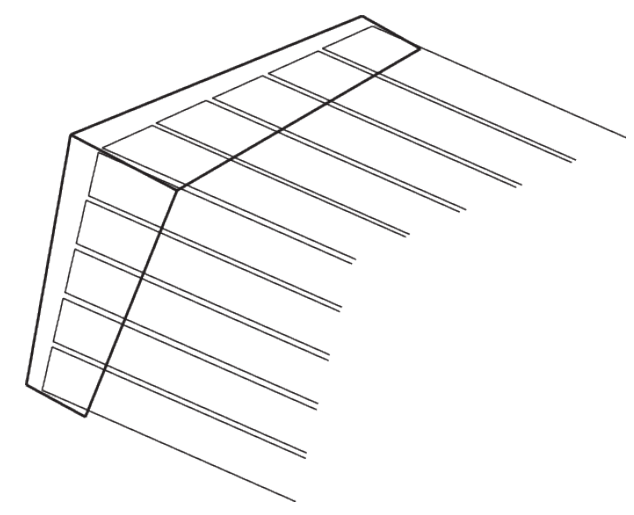

Fig. 1 Discrete horseshoe vortices applied to a swept wing as used in the P-S method.

At each control point, the total velocity vector due to the freestream velocity and the vortex system can be determined from

$$
\boldsymbol{V}_{i}=\boldsymbol{V}_{\boldsymbol{\infty}_{\boldsymbol{i}}}+\sum_{j=1}^{N} \Gamma_{j} \boldsymbol{v}_{\boldsymbol{j} \boldsymbol{i}}
$$

where $\Gamma_{j}$ is the strength of horseshoe vortex $j$ and $v_{j i}$ is the velocity vector induced at control point $i$ by vortex $j$, assuming unit vortex strength. The induced velocity is calculated using the Biot-Savart law [3] and is given by

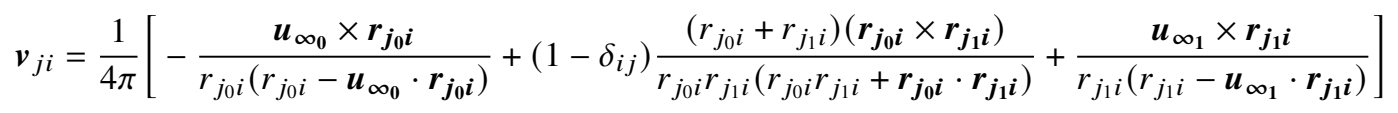

where $\delta_{i j}$ is the Kronecker delta function and $\boldsymbol{r}_{j i}$ is the vector from control point $i$ to a node of horseshoe vortex $j$, with the subscripts 0 and 1 denoting the inbound and outbound vortex nodes, respectively. The inbound and outbound trailing vortex segments are specified by recognizing that the strength of a vortex filament cannot change along its length. Therefore following the right hand convention, one trailing segment points towards the wing, and the other points away from the wing.

The lifting-line hypothesis is then imposed at a control point on each bound vortex segment to find the strength of each horseshoe vortex. Once the strength of each horseshoe vortex is known, the total force acting on the wing can be 
determined by vector summing the forces acting on each section. This will provide an estimate for the lift and induced drag acting on the wing. Sectional moments and parasitic drag can also be included by determining the respective coefficients for each section at the angle of attack found from $\boldsymbol{V}_{\boldsymbol{i}}$ and then summing across the wing.

By including the velocity induced by the bound vortex segment, the P-S method can be used to model more complex wing geometries, such as wing-tail combinations [3]. However, taking the limit as the number of horseshoe vortices goes to infinity, the P-S method can be shown to be the numerical equivalent to Prandtl's original theory for a straight wing [5]. Because of this, the singularities described previously are present in the P-S method, and it fails to grid-converge for swept wings or wings in sideslip [5]. Experience has shown that the P-S method will attempt to force the lift distribution to zero at points of nonzero curvature on the wing in order to avoid predicting infinite lift.

\section{B. General Numerical Lifting-Line Method}

Recently, Reid and Hunsaker presented what they termed a general numerical lifting-line method(herein referred to as the R-H method), which comprises several modifications made to the P-S method to allow for wings with sweep or in sideslip [5]. Among these modifications, Reid and Hunsaker defined an effective LAC (they also place the lifting-line on the wing LAC) for each control point, which has zero curvature at the control point [5]. When calculating the velocity induced at each control point by the vortex system, the vortex system geometry is defined using the effective LAC for the control point under consideration, rather than the original LAC of the wing.

Reid and Hunsaker also added joints to the trailing vortex segments and forced these jointed portions to be perpendicular to the effective LAC [5]. Figure 2 shows these modifications applied to a swept wing.

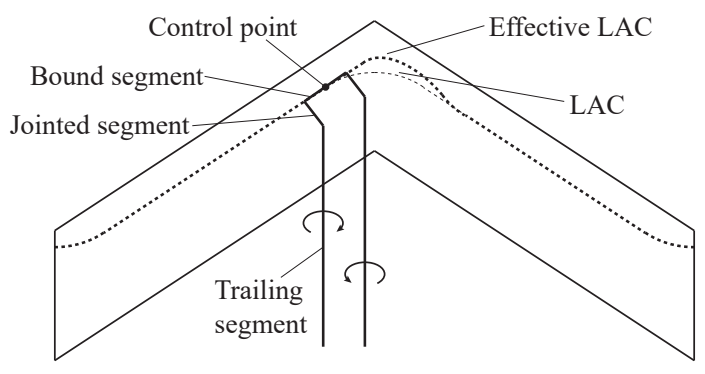

Fig. 2 The effective LAC and jointed horseshoe vortex for a control point on a swept wing as used in the R-H method.

These changes lead to a new definition of $\boldsymbol{v}_{j i}$

$$
\begin{aligned}
\boldsymbol{v}_{j i}=\frac{1}{4 \pi} & {\left[-\frac{\boldsymbol{u}_{\infty_{0}} \times \boldsymbol{r}_{\boldsymbol{j}_{0} i}^{\prime}}{r_{j_{0} i}^{\prime}\left(r_{j_{0} i}^{\prime}-\boldsymbol{u}_{\infty_{0}} \cdot \boldsymbol{r}_{j_{0} i}^{\prime}\right)}+\frac{\left(r_{j_{0} i}^{\prime}+r_{j_{0} i}\right)\left(\boldsymbol{r}_{\boldsymbol{j}_{0} i}^{\prime} \times \boldsymbol{r}_{j_{0} i}\right)}{r_{j_{0} i}^{\prime} r_{j_{0} i}\left(r_{j_{0} i}^{\prime} r_{j_{0} i}+\boldsymbol{r}_{j_{0} i}^{\prime} \cdot \boldsymbol{r}_{j_{0} i}\right)}\right.} \\
& \left.+\left(1-\delta_{i j}\right) \frac{\left(r_{j_{0} i}+r_{j_{1} i}\right)\left(\boldsymbol{r}_{\boldsymbol{j}_{0} i} \times \boldsymbol{r}_{\boldsymbol{j}_{1} i}\right)}{r_{j_{0} i} r_{j_{1} i}\left(r_{j_{0} i} r_{j_{1} i}+\boldsymbol{r}_{\boldsymbol{j}_{0} i} \cdot \boldsymbol{r}_{\boldsymbol{j}_{1} i}\right)}+\frac{\left(r_{j_{1} i}+r_{j_{1} i}^{\prime}\right)\left(\boldsymbol{r}_{j_{1} i} \times \boldsymbol{r}_{j_{1} i}^{\prime}\right)}{r_{j_{1} i} r_{j_{1} i}^{\prime}\left(r_{j_{1} i} r_{j_{1} i}^{\prime}+\boldsymbol{r}_{j_{1} i} \cdot \boldsymbol{r}_{j_{1} i}^{\prime}\right)}+\frac{\boldsymbol{u}_{\infty_{1}} \times \boldsymbol{r}_{j_{1} i}^{\prime}}{r_{j_{1} i}^{\prime}\left(r_{j_{1} i}^{\prime}-\boldsymbol{u}_{\infty_{1}} \cdot \boldsymbol{r}_{j_{1} i}^{\prime}\right)}\right]
\end{aligned}
$$

where ' denotes that the position vector is from the control point to the joint location. The vortex node locations are defined by the effective LAC for control point $i$.

With these modifications, the R-H method grid converges for wings with sweep and in sideslip. Reid and Hunsaker also provided empirical correction factors to the airfoil section properties to account for the effects of sweep [5]. Compared to experimental data collected by Weber et al., the R-H method produces accurate results for the lift distribution on a swept wing [5, 7]. Reid and Hunsaker also showed that solutions obtained using the R-H method are relatively insensitive to the vortex joint length and effective LAC blending distance above a certain threshold. This shows the R-H method does not require tuning to a specific case in order to be accurate.

\section{Current Scope}

Within the current work, we expand the application of the R-H method to allow for an arbitrary number of wings with fully three-dimensional geometry. We also expand upon Reid and Hunsaker's [5] analysis of various methods for 
determining the effect of sweep on airfoil section properties. When evaluating the usefulness of thin airfoil theory, Reid and Hunsaker assume the chordwise circulation distribution is not a function of sweep and so predict no change in the section lift coefficient (when normalized by the swept chord length) [5, 8]. We relax this assumption and show that reliable predictions of swept section properties can be made from thin-airfoil theory.

In presenting the P-S method, Phillips and Snyder derive a linearized version of the lifting-line equation which results in a system of linear equations that can easily be solved [4]. This linear solution gives reasonably accurate results for wings of high aspect ratio at small angles of attack [3]. Phillips and Snyder also derive a Jacobian matrix for the original lifting-line equation, which can be used in Newton's method to iteratively determine the vortex strengths which exactly satisfy the lifting-line equation [4]. We mirror the solution scheme of the P-S method in the current work and develop a linearized version of the general numerical lifting-line equation. We also determine the Jacobian of the general lifting-line equation to be used in Newton's method.

We will refer to the expanded version of the R-H method described in the current work as the Goates-Hunsaker method $(\mathrm{G}-\mathrm{H})$ for solving the general numerical lifting-line problem. Using the G-H method, we discuss how sensitive the lift distribution is to changing the placement of the lifting-line. Both Phillips and Snyder [3], and Reid and Hunsaker [5] place the lifting-line on the wing LAC. We examine the validity of this and whether the lifting-line should be placed on the LAC or some other set of reference points. We will provide recommendations based on comparisons with experimental data. Because we wish to remove any necessary link between the wing LAC and the lifting-line used in the G-H method, we will refer to the effective LAC introduced by Reid and Hunsaker [5] instead as the effective lifting-line. Based on our findings on where to place the lifting-line, we will then compare results obtained from the $\mathrm{G}-\mathrm{H}$ method to experimental data found in the literature.

\section{Geometric Definitions}

We here give definitions for the effective lifting-line and jointed trailing vortices for an arbitrary number of wings with arbitrary sweep, dihedral, and twist. We also develop expressions for the swept section direction vectors based on the local sweep, dihedral, and twist. Throughout, we make use of the traditional body-fixed aircraft coordinate system.

\section{A. Geometry of Multiple Wings}

In order to analyze multiple, interacting lifting surfaces, we must clarify the effective lifting-line introduced in the $\mathrm{R}-\mathrm{H}$ method. The effective lifting-line serves to enforce zero curvature in the lifting-line local to the control point under consideration [5]. Each control point then "sees" a lifting-line which is slightly different from the actual lifting-line of the wing if the control point belongs to that wing. If there are other wings being analyzed to which the control point under consideration does not belong, then that control point will "see" the original, unchanged lifting-line of the other wings. Therefore, for systems of multiple wings, both the original and effective lifting-line geometries must be dealt with.

\section{B. Vector Definition of the Effective Lifting-Line}

To account for arbitrary wing geometries, it is useful to define the lifting-line and effective lifting-line as vector equations. Within this section, we will arbitrarily assume the lifting-line is placed on the wing locus of quarter-chord points (LQC). How the placement of the lifting-line affects the solutions obtained from the G-H method will be discussed later.

First, we must define the sign conventions for sweep and dihedral, as these will depend on which half of the wing we consider. For both, we say the angle is positive if it results from a right-hand rotation about the corresponding axis (see Figure 3). For sweep angle, $\Lambda$, we say a wing which is swept back has a positive sweep angle on the right half and a negative sweep angle on the left half. This follows the right-hand rule if we consider sweep to be a rotation of the LQC about the $z$-axis. For dihedral angle, $\Gamma$, the convention is similar, following the right-hand rule for a rotation about the $x$-axis. A wing with dihedral has a positive dihedral angle on the right half and a negative dihedral angle on the left half. The twist angle, $\tau$, is not dependent on the wing half but is simply a right-hand rotation about the section z-axis, which is dependent upon dihedral. The sweep, dihedral, and twist are all defined relative to the quarter-chord of the wing.

We also define the span fraction, $s$, as the nondimensional distance along the wing locus of quarter-chord points (LQC) projected into the body $y-z$ plane for a given wing half. This distance is nondimensionalized by dividing it by the wing semispan, $b / 2$. The span fraction, $s$, has its origin at the wing root and is equal to unity at the wing tips. Note we do not use the projected wingspan here to nondimensionalize $s$. Using this definition for $s$, the components of the LQC 


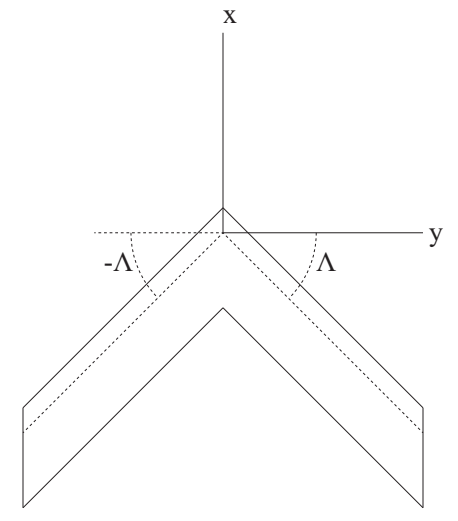

(a) Sweep is positive for a right-hand rotation about the z-axis.

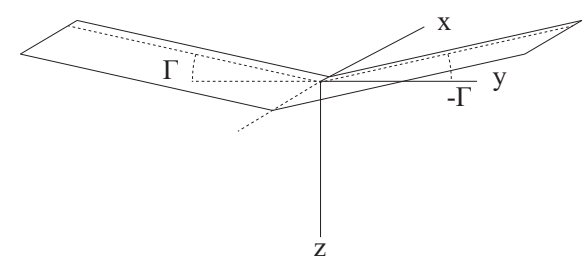

(b) Dihedral is positive for a right-hand rotation about the $\mathrm{x}$-axis.

Fig. 3 Sign conventions for sweep and dihedral.

(relative to the wing root quarter-chord) for the right half of a wing at a given span fraction $s$ are given by

$$
\begin{aligned}
\Delta x_{L Q C} & =-\frac{b}{2} \int_{0}^{s} \tan \Lambda(s) d s \\
\Delta y_{L Q C} & =\frac{b}{2} \int_{0}^{s} \cos \Gamma(s) d s \\
\Delta z_{L Q C} & =\frac{b}{2} \int_{0}^{s} \sin \Gamma(s) d s
\end{aligned}
$$

If the LQC for the left half of the wing is being calculated, then the signs for Eqs. (4) 6) are reversed.

As we move forward in defining the effective lifting-line, we will not take the explicit definition of the lifting-line into consideration. Instead, we simply assume the position of the lifting-line is given by $\boldsymbol{r}_{L L}(s)$ and its spatial derivatives can be determined either analytically or numerically.

To determine the effective lifting-line, $\widetilde{\boldsymbol{r}}_{L L}^{i}$, for a control point $i$, we define a straight line tangent to the original lifting-line at the control point under consideration. This is then blended with the original lifting-line using an exponential blending function in $s$ to obtain

$$
\widetilde{\boldsymbol{r}}_{L L}^{i}(s)=\left(1-e^{-\sigma\left(s-s_{i}\right)^{2}}\right) \boldsymbol{r}_{L L}(s)+e^{-\sigma\left(s-s_{i}\right)^{2}}\left(\boldsymbol{r}_{i}+\left.\frac{d \boldsymbol{r}_{L L}}{d s}\right|_{s=s_{i}}\left(s-s_{i}\right)\right)
$$

where $\sigma$ is a positive, real value and $s_{i}$ is the location of the control point, $\boldsymbol{r}_{i}$, in $s$ (i.e. $\boldsymbol{r}_{i}=\boldsymbol{r}_{L L}\left(s_{i}\right)$ ). In the R-H method [5], $\sigma$ is related to an approximate distance, $\Delta \bar{s}$, (called the blending distance) using the relation

$$
\Delta \bar{s}=\frac{2 \cos \Lambda}{b \sqrt{\sigma}}
$$

We follow this convention in the current work.

\section{Effect of Dihedral and Twist on the Section Direction Vectors}

Beyond affecting the geometry of the lifting-line, the inclusion of dihedral, sweep, and twist affects the definition of the swept section normal, axial, and span vectors used to determine the effective freestream properties and the placement of the trailing vortex joints. Section IV discusses the importance of considering the swept section; within this section, we simply give definitions of the geometry. 
The swept section normal, axial, and span vectors are defined as follows. The swept section span vector, $\boldsymbol{u}_{s_{\Lambda}}$, lies tangential to the lifting-line, oriented such that positive circulation about $\boldsymbol{u}_{s_{\Lambda}}$ produces positive lift (e.g. for a straight wing, $\boldsymbol{u}_{s_{\Lambda}}$ points along the body y-axis). If $\boldsymbol{r}_{L L}$ is known, then $\boldsymbol{u}_{s_{\Lambda}}$ is given by

$$
\boldsymbol{u}_{s_{\Lambda}}=\frac{\boldsymbol{T}}{\|\boldsymbol{T}\|}
$$

where

$$
\boldsymbol{T}=\frac{d \boldsymbol{r}_{L L}}{d t}
$$

where $t$ is defined as displacement along $\boldsymbol{r}_{L L}$ (note this is different from the variable $s$ defined previously). To preserve the sign convention for lift due to circulation, $t$ should increase from left to right along the wing. By normalizing the swept section span vector with respect to its $y$ and $z$ components, $\frac{d \boldsymbol{r}_{L L}}{d s}$ (used in calculating the effective lifting-line) can be determined.

The swept section axial vector, $\boldsymbol{u}_{a_{\Lambda}}$, lies normal to the lifting-line in the plane of the wing. This plane is spanned by $\boldsymbol{u}_{s_{\Lambda}}$ and $\boldsymbol{u}_{a}$, the unswept section axial vector. Using the local dihedral and twist angles, $\boldsymbol{u}_{a}$ is defined as

$$
\boldsymbol{u}_{a}=[-\cos \tau,-\sin \tau \sin \Gamma, \sin \tau \cos \Gamma]^{T}
$$

We must observe that there are two vectors which will satisfy the given definition for $\boldsymbol{u}_{a_{\Lambda}}$, both parallel but opposite in direction. We therefore choose the one which will point most nearly in the direction of $\boldsymbol{u}_{a}$. This can be written as the constraint $\boldsymbol{u}_{a_{\Lambda}} \cdot \boldsymbol{u}_{a}>0$. We can neglect cases where this dot product is equal to zero, as this would only occur when the sweep angle is $\pm 90^{\circ}$. Applying this constraint, the swept section axial vector is given by

$$
\boldsymbol{u}_{a_{\Lambda}}=c_{1} \boldsymbol{u}_{a}+c_{2} \boldsymbol{u}_{s_{\Lambda}}
$$

where

$$
\begin{gathered}
c_{1}=+\sqrt{\frac{1}{1-k^{2}}} \\
c_{2}=-c_{1} k \\
k=\boldsymbol{u}_{s_{\Lambda}} \cdot \boldsymbol{u}_{a}
\end{gathered}
$$

The swept section normal vector is then simply the vector which completes a right-handed, orthonormal system with $\boldsymbol{u}_{a_{\Lambda}}$ and $\boldsymbol{u}_{s_{\Lambda}}$ and is given by

$$
\boldsymbol{u}_{n_{\Lambda}}=\boldsymbol{u}_{a_{\Lambda}} \times \boldsymbol{u}_{s_{\Lambda}}
$$

When determining the effective flow properties at each control point, the section direction vectors should be calculated based on the original lifting-line of the wing, $\boldsymbol{r}_{L L}$. However, when using $\boldsymbol{u}_{a_{\Lambda}}$ to place the vortex joints, the effective lifting-line for each control point should be used.

In certain cases, one may desire to place the lifting-line on the wing LAC, rather than the wing LQC. Often, we assume the LAC and the LQC are coincident. This assumption is appropriate for straight wings. However, for wings with constant sweep, the LAC is usually offset from the LQC using a hyperbolic interpolation suggested by Küchemann. We suggest that, if used, Küchemann's correction should be applied in the direction of $\boldsymbol{u}_{a}$.

\section{Placement of the Vortex Joints}

Reid specifies that the jointed portions of the trailing vortices lie in the plane of the wing, normal to the lifting-line [5]. To satisfy this, the jointed vortex segments are aligned with $\boldsymbol{u}_{a_{\Lambda}}$ evaluated at the vortex node location. The joint location is then given by

$$
\widetilde{\boldsymbol{r}}_{j}^{\prime}=\widetilde{\boldsymbol{r}}_{L L_{j}}+\delta c_{j} \boldsymbol{u}_{a_{\Lambda} j}
$$


where $\delta$ is the vortex joint length given as a fraction of the wing chord and $c_{j}$ is the local chord length at the vortex node location. Note that the position of the effective lifting-line is used in eq. (17), rather than the true lifting-line. This is because the jointed vortices are part of calculating the induced velocity at each control point, for which the effective lifting-line must be used. In determining the joint locations, $\boldsymbol{u}_{a_{\Lambda}}$ should also be calculated using the effective lifting-line.

Experience has shown that an issue arises when defining the vortex joint locations using $\boldsymbol{u}_{a_{\Lambda}}$ for wings with sudden changes in twist. Since $\boldsymbol{u}_{a_{\Lambda}}$ is defined using $\boldsymbol{u}_{a}$, which is dependent upon the local twist, a sudden change in twist results in a sudden change in the direction of $\boldsymbol{u}_{a_{\Lambda}}$ as a function of span. This sudden change in direction amounts to a discontinuity in the trailing vortex sheet, which can cause large spikes in local induced velocity near the discontinuity. This tends to negatively affect solver convergence. To remedy this, we recommend blending $\boldsymbol{u}_{a_{\Lambda}}$ in the manner of Eq. (7). In doing this, $\boldsymbol{u}_{a_{\Lambda}}$ at control point $i$ is analogous to the 'straight' portion of Eq. (7), and the swept section axial vectors at the vortex nodes are analogous to the 'true' portion of Eq. (7). Implementing this blending strategy results in greatly improved solver performance and much more reasonable induced velocities while having negligible impact on the final circulation distribution.

\section{Properties of Swept Wing Sections}

Within potential flow, the basis of many prediction methods is to replace the wing geometry with a set of elementary flows (typically doublet or source sheets in a freestream) of specified strength such that the surface of the wing becomes a stream surface of the flow. In the case of an infinite swept wing (i.e. a swept airfoil section) an alternate coordinate system is typically defined which has been rotated by the sweep angle about the $z$-axis so the rotated $y$-axis is then parallel to the wing. We will call the rotated $y$-axis the $\eta$-axis and the rotated $x$-axis the $\xi$-axis. We can then express the freestream velocity acting on the wing in either the $x-y-z$ system or the $\xi-\eta-z$ system.

$$
\boldsymbol{V}_{\infty}=u \boldsymbol{i}_{x}+v \boldsymbol{i}_{y}+w \boldsymbol{i}_{z}=v_{\xi} \boldsymbol{i}_{\xi}+v_{\eta} \boldsymbol{i}_{\eta}+v_{z} \boldsymbol{i}_{z}
$$

If we assume the wing is of constant cross-section, then the component of freestream velocity parallel to the wing, $v_{\eta}$, will automatically satisfy the requirement that the surface of the wing be a stream surface of the flow [9]. This means that the arrangements and strengths of the elementary flows used to simulate the wing geometry will not be affected by $v_{\eta}$. We thus assume that $v_{\eta}$ should be ignored when calculating the aerodynamics of a swept wing using potential flow theory. This assumption is made by many others (see, for example, [9-11]).

Lifting-line theory differs from other potential flow methods in that, instead of solving for source or vortex strengths to satisfy a streamline condition, the vortex strengths are determined such that each bound segment produces the same circulation as an infinite wing of the same geometry at the same angle of attack. We replace the wing with a set of jointed horseshoe vortices, their bound portions forming a lifting-line. The bound vortex segment will then only induce velocity, and thereby circulation, in the plane normal to the lifting-line. Thus, if we desire to equate the bound vortex segment to an airfoil section, that section must also only induce circulation in the plane normal to the lifting-line. Thus, we assume we must evaluate the aerodynamic properties of the wing section which lies in the plane normal to the lifting-line.

Since we are concerned with the wing section in the plane normal to the lifting-line, we define a section sweep angle, $\Lambda_{i}$. We define the section sweep angle as the minimum angle between the body $y-z$ plane and a line tangent to the lifting-line at the control point under consideration. This angle is given by

$$
\Lambda_{i}=-\arctan \left(\frac{d \boldsymbol{r}_{L L}\left(s_{i}\right)}{d s}\right)
$$

Note this follows the sign convention for sweep described previously.

We can observe that using the vortex lifting law to predict lift automatically takes sweep into account through use of a cross-product [3]. This law is given by

$$
d \boldsymbol{F}=\rho \Gamma \boldsymbol{V} \times \boldsymbol{d l}
$$

However, we must use other means to account for the change in lift of a swept wing section. We will discuss how the changes to the aerodynamics of swept sections are accounted for in the G-H method using an effective freestream velocity (following from our discussion on the effect of $v_{\eta}$ ) and an effective section geometry (following from our discussion on section circulation). The definition of an effective section angle of attack follows from the effective freestream velocity. We will then discuss how swept section aerodynamic coefficients are to be redimensionalized and how the change in these coefficients can be predicted. 


\section{A. Effective Section Geometry}

As discussed previously, when evaluating the section aerodynamics of a swept wing, it is the section in the plane normal to the lifting-line which should be considered. Since sweep is traditionally defined as a shear transformation, the section geometry is usually known in the plane normal to the unswept spanwise direction. For a wing of constant cross-section, the swept section geometry is then simply the unswept section geometry scaled in the chordwise direction by $\cos \Lambda_{i}$. Because of this, the swept chord length is given by

$$
c_{i_{\Lambda}}=c_{i} \cos \Lambda_{i}
$$

This scaling of the section geometry is approximate for wings of non-constant cross-section; as long as there are not rapid changes in section geometry as a function of span, the errors introduced by this approximation will be small.

\section{Effective Flap Deflection}

The change in section geometry due to sweep also has an effect on the flap deflection in the plane normal to the lifting-line as shown in Fig. 4 . For a flap with a given unswept section geometry and flap deflection, we can determine the effective swept flap deflection, $\delta_{f_{\Lambda}}$, from the unswept flap deflection and the local sweep angle. We do this by recognizing the vertical distance from the chord line to the tip of the flap, $h_{f}$, does not change with sweep. We can thus equate
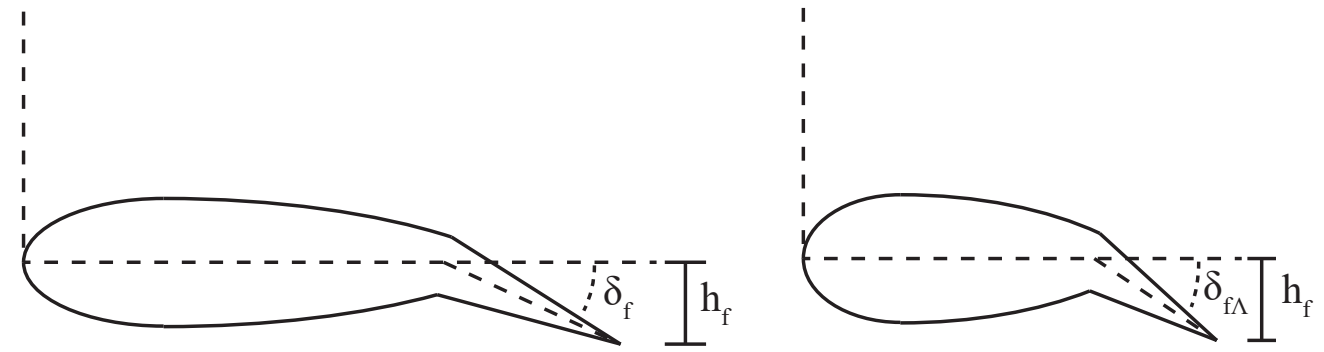

Fig. 4 The effect of sweep on section flap deflection angle. The left image is unswept, the right image is swept.

$$
c_{f} \sin \delta_{f}=c_{f_{\Lambda}} \sin \delta_{f_{\Lambda}}
$$

Note that $c_{f}$ and $c_{f_{\Lambda}}$ are related in the same way as $c$ and $c_{\Lambda}$. Solving for $\delta_{f_{\Lambda}}$, we obtain

$$
\delta_{f_{\Lambda}}=\sin ^{-1}\left(\cos \Lambda \sin \delta_{f}\right)
$$

This has an effect on the corrections to section coefficients discussed subsequently.

\section{B. Effective Freestream Velocity}

As shown previously, when determining the aerodynamics of a swept, infinite wing, the freestream velocity parallel to the wing, $v_{\eta}$, should be ignored. We thus define the effective freestream velocity, $V_{\infty_{\Lambda}}$, as the component of the freestream velocity vector in the plane normal to the lifting-line.

We can obtain the effective freestream vector by projecting the freestream vector into the plane normal to the lifting-line. Recognizing that this plane is the orthogonal complement to $\operatorname{span}\left\{\boldsymbol{u}_{s_{\Lambda}}\right\}$, the projection matrix that will project a vector into the plane normal to the lifting-line is

$$
P_{\mathrm{eff}}=I_{3 \times 3}-\boldsymbol{u}_{s_{\Lambda}} \boldsymbol{u}_{s_{\Lambda}}^{T}
$$

The effective freestream velocity is then given by

$$
\boldsymbol{V}_{\infty_{\Lambda}}=P_{\mathrm{eff}} \boldsymbol{V}_{\infty}
$$


Within the G-H method, we also are concerned with the change in local dynamic pressure caused by induced velocities. However, since we are still only concerned with the component of velocity acting in the plane normal to the lifting-line, we use $P_{\text {eff }}$ to determine the effective local velocity, $\boldsymbol{V}_{\Lambda_{i}}$, given by

$$
\boldsymbol{V}_{\Lambda_{i}}=P_{\mathrm{eff}} \boldsymbol{V}_{i}
$$

\section{Swept Section Angle of Attack}

Using the effective velocity (either freestream or local), we define the swept section angle of attack as

$$
\alpha_{\Lambda}=\arctan \left(\frac{\boldsymbol{V}_{\Lambda} \cdot \boldsymbol{u}_{n_{\Lambda}}}{\boldsymbol{V}_{\Lambda} \cdot \boldsymbol{u}_{a_{\Lambda}}}\right)
$$

The swept angle of attack is then used to determine the section lift and moment coefficients.

\section{Swept Section Reynolds and Mach Number}

One significant advantage of numerical lifting-line theory over other potential flow methods is the possibility for correcting section aerodynamic coefficients based on Mach and Reynolds number. The change in effective velocity for a swept section also results in a change in the section Mach and Reynolds number. For a swept section, the effective Mach number is given by

$$
M_{\Lambda}=\frac{\left|V_{\Lambda}\right|}{a}
$$

where $a$ is the local speed of sound. Since Reynolds number is a function of both the flow velocity and the reference length, the Reynolds number is affected in two ways by sweep. As discussed previously, the chord length of a swept section is reduced by the cosine of the section sweep angle, $\Lambda_{i}$. Thus, the effective Reynolds number is given by

$$
\operatorname{Re}_{\Lambda}=\frac{\left|\boldsymbol{V}_{\Lambda}\right| c_{\Lambda}}{v}
$$

\section{Redimensionalization of Swept Section Coefficients}

As sweep has an effect on the section geometry and freestream properties, we would expect a change in how section aerodynamic coefficients are redimensionalized. In correcting section aerodynamic coefficients for sweep, we will assume the nondimensional coefficients are given with respect to a unit chord length. We would then redimensionalize using the swept chord length, $c_{\Lambda}$. However, to obtain the aerodynamic force for a discrete section of the wing, we must multiply by the area of the entire section. Where sweep creates a decrease in section chord length by cos $\Lambda$, it also creates an increase in the length of the segment in the directions of $\boldsymbol{u}_{s_{\Lambda}}$ by $1 / \cos \Lambda$. Thus, the area of the swept discrete section, $d S$, remains the same as in the unswept case. Using the effective section velocity to redimensionalize the coefficient, the aerodynamic force produced by a section is given by

$$
d F=\frac{1}{2} \rho\left|V_{\Lambda}\right|^{2} \widetilde{C}_{F_{\Lambda}} d S
$$

To redimensionalize the section moment, we include $c_{\Lambda}$ as the required reference length.

$$
d M=\frac{1}{2} \rho\left|V_{\Lambda}\right|^{2} \widetilde{C}_{m_{\Lambda}} c_{\Lambda} d S
$$

As a departure from both the P-S [4] and R-H [5] methods, we have chosen to redimensionalize the swept section lift coefficient using the effective local velocity at each control point. Within the P-S [4] and R-H methods [5], the swept section lift coefficient is redimensionalized using the freestream velocity to allow for nondimensionalization of Eq. (38) [4, 5]. However, doing so reduces the accuracy of the final solution, as this neglects the increase in dynamic pressure seen by the wing due to induced velocity. 


\section{Corrections to Section Properties Using Thin-Airfoil Theory}

The change in lift on an infinite wing due to sweep predicted by thin airfoil theory has been considered before. Rosen and Rand [10] describe how, under the assumptions of thin airfoil theory, the lift per unit span on an infinite swept wing is reduced due to a decrease in the effective angle of attack. They show that, assuming small angles, the effective angle of attack is given by

$$
\alpha_{\Lambda}=\alpha \cos \Lambda
$$

Note that this relation can be obtained by applying the small angle approximation to Eq. [27). Rosen and Rand [10] take the lift slope as being $2 \pi$ (as predicted by thin airfoil theory) but only consider a symmetric airfoil (i.e. $\alpha_{L 0}=0$ ). We can expand this, however, to provide a correction for asymmetric swept airfoils based on thin airfoil theory.

Thin airfoil theory models an airfoil section as an infinite vortex sheet lying on the chord (or camber) line of the section [2, 3]. The vortex strength per unit distance along the sheet, $\gamma(x)$, is then solved for such that the camber line of the airfoil becomes a streamline of the flow and the Kutta condition $(\gamma(c)=0)$ is satisfied. Thin airfoil theory predicts a lift slope of $2 \pi$ regardless of section geometry. Thus, for a symmetric airfoil, the swept section lift coefficient predicted by thin airfoil theory is no different than the unswept section lift coefficient, given the effective angle of attack is used. From thin airfoil theory, the zero-lift angle of attack is predicted to be

$$
\alpha_{L 0}=-\frac{1}{\pi} \int_{0}^{\pi} \frac{d y_{c}}{d x}(1-\cos \theta) d \theta
$$

where

$$
x=\frac{c}{2}(1-\cos \theta)
$$

and $y_{c}(x)$ is the equation of the airfoil camber line [12]. If we assume the chord length has been normalized for a given swept airfoil, then sweep will have no effect on the lift slope predicted by thin airfoil theory. However, Eq. (33) shows $\alpha_{L 0}$ is dependent on the geometry of the camber line through $\frac{d y_{c}}{d x}$, which does change with section sweep angle. When sweep is applied, $\frac{d y_{c}}{d x}$ will be scaled by a constant factor of $1 / \cos \Lambda$. Since this is a constant, it can be taken out of the integral in Eq. 33, , yielding

$$
\alpha_{L 0_{\Lambda}}=-\frac{1}{\pi \cos \Lambda} \int_{0}^{\pi} \frac{d y_{c}}{d x}(1-\cos \theta) d \theta=\frac{\alpha_{L 0}}{\cos \Lambda}
$$

Thus, for a cambered airfoil, sweep creates a change in the lift coefficient at a given angle of attack due to a change in zero-lift angle of attack. The correction given by Eq. (35) makes no assumption as to the magnitude of the local sweep and so may be applied to arbitrary wing geometries as long as the assumptions of thin-airfoil theory are still valid.

Similar to the effect of sweep on the section lift coefficient, thin airfoil theory predicts a change in section moment coefficient due to sweep. From thin airfoil theory, the section moment coefficient about the quarter-chord is given by

$$
\widetilde{C}_{m_{c / 4}}=\frac{1}{2} \int_{0}^{\pi} \frac{d y_{c}}{d x}(\cos (2 \theta)-\cos \theta) d \theta
$$

This is constant with respect to angle of attack. As with zero-lift angle of attack, sweep scales the camber line derivative by $1 / \cos \Lambda$. This factor can be taken out of the integral, yielding

$$
\widetilde{C}_{m_{c / 4} \Lambda}=\frac{\widetilde{C}_{m_{c / 4}}}{\cos \Lambda}
$$

\section{E. Corrections to Viscous Section Drag Coefficient}

One of the major advantages of numerical lifting-line theory, compared to other potential flow methods, is the ability to account for some viscous effects through the evaluation of the parasitic drag on each airfoil section based on a knowledge of the freestream properties. However, determining the section drag coefficient on swept wings poses unique challenges and must be treated differently than swept lift and moment coefficients. Parasitic drag is a viscous phenomenon dominated by boundary layer growth in the streamwise direction. Because of this, the assumptions made previously to neglect $v_{\eta}$ and to consider the geometry of the wing in the plane normal to the lifting-line are not valid when considering parasitic drag. 
Different methods for predicting the viscous section drag coefficient of a swept wing have been suggested in the past. The P-S method uses the unswept section drag coefficient at the unswept angle of attack and applies that drag force in the direction of the local velocity [3]. However, this fails to take into account spanwise boundary layer development which occurs on swept wings. A "2.5D" method has been proposed which uses CFD and somewhat accounts for spanwise flow development [13, 14]. With this method, the drag coefficient is determined as a function of sweep and angle of attack for a given section geometry using a CFD solution over an infinite swept wing. This drag coefficient can then be used in a lifting-line solution based on the local unswept angle of attack. However, this is not a correction, per se, as the swept section drag coefficient is calculated entirely independent of the unswept section drag coefficient.

Within the G-H method, we desire to estimate the parasitic drag of a swept wing based only on the parasitic drag on an unswept wing of the same section geometry. As a first-order approach, we mimic the P-S method [3] within the G-H method, as this does not require any knowledge of the actual boundary layer behavior over the swept wing. We acknowledge this has limited accuracy, but is desirable for simplicity and ease of implementation. Corrections to viscous predictions for swept wings is an area of possible future research.

\section{Solution Methods}

The governing lifting-line equation is derived by recognizing that the only aerodynamic force produced in potential flow is lift due to circulation. This means the aerodynamic force predicted by the vortex lifting law for a given bound vortex segment must be equal to the section lift predicted by the aerodynamic properties of the corresponding swept wing section. This relation can be expressed as

$$
\rho \Gamma_{i}\left|\boldsymbol{V}_{\Lambda_{i}} \times d \boldsymbol{l}_{i}\right|=\frac{1}{2} \rho\left|\boldsymbol{V}_{\Lambda_{i}}\right|^{2} \widetilde{C}_{L_{\Lambda} i}(\boldsymbol{a}) d S_{i}
$$

where $\boldsymbol{a}$ is the set of local aerodynamic parameters, such as angle of attack, flap deflection, Reynolds number, etc. and $d \boldsymbol{l}_{i}$ is the vector extending from the inbound vortex node to the outbound vortex node for horseshoe vortex $i$. Note that the vortex lift in Eq. (38) is calculated using the effective local velocity, rather than the total local velocity. It can be shown that these are equivalent, as the cross product accounts for the decrease in lift due to non-perpendicularity if the total local velocity is used. We have chosen to use the effective local velocity for simplicity in deriving later equations.

Equation (38) follows the standard definition of lift in potential flow and assumes positive circulation generates positive lift. This can be expressed mathematically as

$$
\operatorname{sign}\left(\Gamma_{i}\right)=\operatorname{sign}\left(\widetilde{C}_{L i}\right)
$$

Since $d \boldsymbol{l}_{i}$ specifies the direction of the bound vortex segment, $d \boldsymbol{l}_{i}$ is defined such that Eq. (39) holds. Thus, the horseshoe vortex nodes are placed so as to proceed from the left tip to the right tip of each lifting surface. This will result in $d \boldsymbol{l}_{i}$ having the same direction as $\boldsymbol{u}_{S_{\Lambda}}$.

Simplifying, Eq. (38) can be written as

$$
2\left|\boldsymbol{V}_{\Lambda_{i}} \times \boldsymbol{d} l_{i}\right| \Gamma_{i}-\left|\boldsymbol{V}_{\Lambda_{i}}\right|^{2} \widetilde{C}_{L_{\Lambda} i}(\boldsymbol{a}) d S_{i}=0
$$

Equation (40) is the governing equation of the G-H method. To determine the aerodynamics of the given wing configuration, all that is required is to determine the strength of each horseshoe vortex such that Eq. (40) is satisfied at each control point. We will now delineate two solution methods for determining this vortex strength distribution.

\section{A. Linearized Solution}

As a fast, first approximation, a linearized version of Eq. 40] can be used to solve for the vortex strength distribution. By applying the small-angle approximation and dropping nonlinear terms, the lifting-line equation can be written as a set of linear equations. At this point in the derivation, for convenience, we will drop the $i$ subscript, denoting evaluation at a control point, and assume this is implied.

To obtain a linear system of equations, we begin by assuming a linear model for the swept section lift coefficient.

$$
\widetilde{C}_{L_{\Lambda}}(\boldsymbol{a}) \approx \widetilde{C}_{L_{\Lambda}, \alpha}\left(\alpha_{\Lambda}-\alpha_{L 0_{\Lambda}}+\varepsilon \delta_{f_{\Lambda}}\right)
$$

where the swept angle of attack, $\alpha_{\Lambda}$, is given by Eq. 27p and where $\varepsilon$ and $\delta_{f_{\Lambda}}$ are the section flap efficiency and swept deflection, respectively. The swept section lift slope and zero-lift angle of attack are determined from the relations described in Section IV] We ignore any influence of the Mach and Reynolds numbers on the lift coefficient. 
Assuming small angles of attack, the induced velocities will be small compared to the freestream velocity and the effective freestream will be approximately aligned with the swept axial vector. The following approximation can then be applied to the denominator in Eq. 27]

$$
\boldsymbol{V}_{\Lambda} \cdot \boldsymbol{u}_{\boldsymbol{a}_{\Lambda}} \approx \boldsymbol{V}_{\infty_{\Lambda}} \cdot \boldsymbol{u}_{\boldsymbol{a}_{\boldsymbol{\Lambda}}} \approx\left|\boldsymbol{V}_{\infty_{\Lambda}}\right|
$$

Assuming small angles, we can also apply the approximation $\tan x \approx x$. The local swept angle of attack can then be approximated as

$$
\alpha_{\Lambda} \approx \boldsymbol{u}_{\infty_{\Lambda}} \cdot \boldsymbol{u}_{\boldsymbol{n}_{\Lambda}}+\frac{1}{\left|\boldsymbol{V}_{\infty_{\Lambda}}\right|}\left(\sum_{j=1}^{N} \Gamma_{j} \boldsymbol{v}_{j i}\right) \cdot \boldsymbol{u}_{\boldsymbol{n}_{\Lambda}}
$$

where

$$
u_{\infty_{\Lambda}} \equiv \frac{V_{\infty_{\Lambda}}}{\left|V_{\infty_{\Lambda}}\right|}
$$

To further linearize Eq. (40), we can write the magnitude of the effective local velocity as

$$
\begin{aligned}
\left|\boldsymbol{V}_{\Lambda}\right|^{2} & =P_{\mathrm{eff}}\left(\boldsymbol{V}_{\infty}+\sum_{j=1}^{N} \Gamma_{j} \boldsymbol{v}_{\boldsymbol{j} \boldsymbol{i}}\right) \cdot P_{\mathrm{eff}}\left(\boldsymbol{V}_{\infty}+\sum_{j=1}^{N} \Gamma_{j} \boldsymbol{v}_{\boldsymbol{j} \boldsymbol{i}}\right) \\
& =\left|\boldsymbol{V}_{\infty_{\Lambda}}\right|^{2}+2 \boldsymbol{V}_{\infty_{\Lambda}} \cdot P_{\mathrm{eff}}\left(\sum_{j=1}^{N} \Gamma_{j} \boldsymbol{v}_{\boldsymbol{j} \boldsymbol{i}}\right)+P_{\mathrm{eff}}\left(\sum_{j=1}^{N} \Gamma_{j} \boldsymbol{v}_{\boldsymbol{j} \boldsymbol{i}}\right) \cdot P_{\mathrm{eff}}\left(\sum_{j=1}^{N} \Gamma_{j} \boldsymbol{v}_{\boldsymbol{j} \boldsymbol{i}}\right)
\end{aligned}
$$

The last term in Eq. (45) is neglected, as it is second-order in the induced velocities, which we assume to be small. As an approximation at small angles of attack, we can also assume the induced velocities, $\Gamma_{j} \boldsymbol{v}_{\boldsymbol{j} i}$, are nearly perpendicular to the effective freestream, which makes the second term go to zero. $\left|\boldsymbol{V}_{\Lambda}\right|^{2}$ can then be approximated as $\left|\boldsymbol{V}_{\infty_{\Lambda}}\right|^{2}$. Applying all the approximations thus far to the right half of Eq. [40, we can write

$$
\left|\boldsymbol{V}_{\boldsymbol{\Lambda}}\right|^{2} \widetilde{C}_{L_{\Lambda}}(\boldsymbol{a}) d S \approx\left|\boldsymbol{V}_{\infty_{\Lambda}}\right|^{2} \widetilde{C}_{L_{\Lambda}, \alpha}\left(\boldsymbol{u}_{\infty_{\Lambda}} \cdot \boldsymbol{u}_{\boldsymbol{n}_{\Lambda}}+\frac{1}{\left|\boldsymbol{V}_{\infty_{\Lambda}}\right|} \sum_{j=1}^{N} \Gamma_{j} \boldsymbol{v}_{\boldsymbol{j} \boldsymbol{i}} \cdot \boldsymbol{u}_{\boldsymbol{n}_{\Lambda}}-\alpha_{L 0_{\Lambda}}+\varepsilon \delta_{f_{\Lambda}}\right) d S
$$

If we drop the nonlinear terms from the first term in Eq. (40) (we assume these are small at small angles of attack) and combine this with Eq. (46), we obtain

$$
2\left|\boldsymbol{u}_{\infty_{\Lambda} i} \times \boldsymbol{d} \boldsymbol{l}_{\boldsymbol{i}}\right| \Gamma_{i}-\widetilde{C}_{L_{\Lambda}, \alpha_{i}}\left(\sum_{j=1}^{N} \Gamma_{j} \boldsymbol{v}_{\boldsymbol{j} \boldsymbol{i}} \cdot \boldsymbol{u}_{\boldsymbol{n}_{\boldsymbol{\Lambda}} \boldsymbol{i}}\right) d S_{i}=\left|\boldsymbol{V}_{\infty_{\Lambda} \boldsymbol{i}}\right| \widetilde{C}_{L_{\Lambda}, \alpha_{i}}\left(\boldsymbol{u}_{\infty_{\Lambda}} \cdot \boldsymbol{u}_{\boldsymbol{n}_{\boldsymbol{\Lambda}} \boldsymbol{i}}-\alpha_{L 0_{\Lambda} i}+\varepsilon_{i} \delta_{f_{\Lambda} i}\right) d S_{i}
$$

Equation (47) is linear in $\Gamma$ and is readily solved at a set of control points using numerical linear algebra techniques. This solution proves remarkably accurate for lifting surfaces with high aspect ratio and low angle of attack. Experience has shown the norm of the residual vector obtained from substituting the solution for $\Gamma$ from Eq. (47) into Eq. (40) is typically on the order of $10^{-1}$ for a traditional airframe at small angles of attack. This solution can also be used as an initial guess for iteratively solving Eq. (40) using the method described next.

We must quickly remark that the right hand side of Eq. $447 \mathrm{can}$ alternatively be written as $\left|\boldsymbol{V}_{\infty_{\Lambda} i}\right| \widetilde{C}_{L_{\Lambda} i}(\boldsymbol{a}) d S_{i}$ where $\boldsymbol{a}$ represents the airfoil parameters such as angle of attack, Reynolds number, etc. at each section, not taking induced velocity into account. This represents linearizing the system about the current global angle of attack, rather than an angle of attack of zero. This also makes no linearizing assumptions about angle of attack and flap deflection.

\section{B. Nonlinear Improvement Using Newton's Method}

If we write the vortex strengths as a vector, $\boldsymbol{\Gamma}$, we can present Eq. 40 as a vector equation of the form

$$
f(\Gamma)=R
$$


where

$$
f_{i}(\boldsymbol{\Gamma})=2\left|\boldsymbol{V}_{\Lambda_{i}} \times \boldsymbol{d} l_{i}\right| \Gamma_{i}-\left|\boldsymbol{V}_{\Lambda_{i}}\right|^{2} \widetilde{C}_{L_{\Lambda} i}(\boldsymbol{a}) d S_{i}
$$

and $\boldsymbol{R}$ is a vector of residuals. Finding the vortex strengths, $\boldsymbol{\Gamma}$, then simply reduces to finding the roots of Eq. (48). Newton's method lends itself well to this root-finding problem. Given an initial guess for $\boldsymbol{\Gamma}$ and a corresponding residual vector, $\boldsymbol{R}$, the update to $\boldsymbol{\Gamma}$ can be found from Newton's corrector formula

$$
J \Delta \boldsymbol{\Gamma}=-\boldsymbol{R}
$$

where $J$ is the Jacobian matrix of $\boldsymbol{f}(\boldsymbol{\Gamma})$ and $\Delta \boldsymbol{\Gamma}$ is the update. Equation (40) is readily differentiated with respect to $\boldsymbol{\Gamma}$ and so we can derive an expression for $J$ analytically. The elements of $J$ are given by

$$
J_{i j}=\frac{\partial}{\partial \Gamma_{j}}\left(2\left|\boldsymbol{V}_{\Lambda_{i}} \times \boldsymbol{d} \boldsymbol{l}_{\boldsymbol{i}}\right| \Gamma_{i}-\left|\boldsymbol{V}_{\Lambda_{i}}\right|^{2} \widetilde{C}_{L_{\Lambda} i}(\boldsymbol{a}) d S_{i}\right)
$$

As a first step, it is useful to write the derivative of the effective total velocity, $\boldsymbol{V}_{\Lambda_{i}}$ with respect to $\Gamma_{j}$, as this term occurs frequently in subsequent steps.

$$
\frac{\partial}{\partial \Gamma_{j}}\left(\boldsymbol{V}_{\Lambda_{i}}\right)=\frac{\partial}{\partial \Gamma_{j}}\left(P_{\mathrm{eff}}\left(\boldsymbol{V}_{\infty_{i}}+\sum_{j=1}^{N} \Gamma_{j} \boldsymbol{v}_{j i}\right)\right)=P_{\mathrm{eff}} \boldsymbol{v}_{j i}
$$

The derivative of the first term in the right hand side of Eq. (51) depends upon whether $j=i$. For the case where $j=i$, we must use the product rule, which yields:

$$
\frac{\partial}{\partial \Gamma_{j}}\left(2\left|\boldsymbol{V}_{\Lambda_{i}} \times \boldsymbol{d} \boldsymbol{l}_{\boldsymbol{i}}\right| \Gamma_{i}\right)=2\left|\boldsymbol{V}_{\Lambda_{i}} \times \boldsymbol{d} \boldsymbol{l}_{\boldsymbol{i}}\right|+2 \Gamma_{i} \frac{\partial}{\partial \Gamma_{j}}\left(\left|\boldsymbol{V}_{\Lambda_{i}} \times \boldsymbol{d} \boldsymbol{l}_{\boldsymbol{i}}\right|\right)=2\left|\boldsymbol{V}_{\Lambda_{i}} \times \boldsymbol{d} \boldsymbol{l}_{\boldsymbol{i}}\right|+2 \Gamma_{i} \frac{\left(\boldsymbol{V}_{\Lambda_{i}} \times \boldsymbol{d} \boldsymbol{l}_{\boldsymbol{i}}\right) \cdot\left(P_{\mathrm{eff}} \boldsymbol{v}_{j i} \times \boldsymbol{d} \boldsymbol{l}_{\boldsymbol{i}}\right)}{\left|\boldsymbol{V}_{\Lambda_{i}} \times \boldsymbol{d} l_{\boldsymbol{i}}\right|}
$$

For the case where $j \neq i$, the product rule is no longer needed and the first term of Eq. (53) disappears, yielding

$$
\frac{\partial}{\partial \Gamma_{j}}\left(2\left|\boldsymbol{V}_{\Lambda_{i}} \times \boldsymbol{d} l_{\boldsymbol{i}}\right| \Gamma_{i}\right)=2 \Gamma_{i} \frac{\left(\boldsymbol{V}_{\Lambda_{i}} \times \boldsymbol{d} \boldsymbol{l}_{\boldsymbol{i}}\right) \cdot\left(P_{\mathrm{eff}} \boldsymbol{v}_{\boldsymbol{j} \boldsymbol{i}} \times \boldsymbol{d} \boldsymbol{l}_{\boldsymbol{i}}\right)}{\left|\boldsymbol{V}_{\Lambda_{i}} \times \boldsymbol{d} \boldsymbol{l}_{\boldsymbol{i}}\right|}
$$

The second term in Eq. 51] must be evaluated using the product rule

$$
\frac{\partial}{\partial \Gamma_{j}}\left(-\left|\boldsymbol{V}_{\Lambda_{i}}\right|^{2} \widetilde{C}_{L i}(\boldsymbol{a}) d S_{i}\right)=-d S_{i}\left[\left|\boldsymbol{V}_{\Lambda_{i}}\right|^{2} \frac{\partial \widetilde{C}_{L_{\Lambda} i}}{\partial \Gamma_{j}}+\widetilde{C}_{L_{\Lambda} i} \frac{\partial}{\partial \Gamma_{j}}\left(\left|\boldsymbol{V}_{\Lambda_{i}}\right|^{2}\right)\right]
$$

We assume the section lift coefficient is dependent upon $\Gamma_{j}$ through the effective angle of attack, Reynolds number, and Mach number. We thus write

$$
\frac{\partial \widetilde{C}_{L_{\Lambda} i}}{\partial \Gamma_{j}}=\widetilde{C}_{L_{\Lambda}, \alpha_{i}} \frac{\partial \alpha_{\Lambda_{i}}}{\partial \Gamma_{j}}+\widetilde{C}_{L_{\Lambda}, R e_{i}} \frac{\partial R e_{i}}{\partial \Gamma_{j}}+\widetilde{C}_{L_{\Lambda}, M_{i}} \frac{\partial M_{i}}{\partial \Gamma_{j}}
$$

The derivatives of section lift with respect to angle of attack, Reynolds number, and Mach number are assumed to be known airfoil properties. The swept section angle of attack at any given control point is given by Eq. 27]. Taking the derivative of this with respect to $\Gamma_{j}$, we obtain

$$
\frac{\partial \alpha_{\Lambda_{i}}}{\partial \Gamma_{j}}=\frac{\left(\boldsymbol{V}_{\Lambda_{i}} \cdot \boldsymbol{u}_{\boldsymbol{a}_{\boldsymbol{\Lambda}} \boldsymbol{i}}\right)\left(P_{\mathrm{eff}} \boldsymbol{v}_{\boldsymbol{j} \boldsymbol{i}} \cdot \boldsymbol{u}_{\boldsymbol{n}_{\boldsymbol{\Lambda}} \boldsymbol{i}}\right)-\left(\boldsymbol{V}_{\Lambda_{i}} \cdot \boldsymbol{u}_{\boldsymbol{n}_{\boldsymbol{\Lambda}} \boldsymbol{i}}\right)\left(P_{\mathrm{eff}} \boldsymbol{v}_{\boldsymbol{j} \boldsymbol{i}} \cdot \boldsymbol{u}_{\boldsymbol{a}_{\boldsymbol{\Lambda}} \boldsymbol{i}}\right)}{\left(\boldsymbol{V}_{\Lambda_{i}} \cdot \boldsymbol{u}_{\boldsymbol{a}_{\boldsymbol{\Lambda}} \boldsymbol{i}}\right)^{2}+\left(\boldsymbol{V}_{\Lambda_{i}} \cdot \boldsymbol{u}_{\boldsymbol{n}_{\boldsymbol{\Lambda}} \boldsymbol{i}}\right)^{2}}
$$

The swept section Reynolds number at any given control point is equal to

$$
R e_{i}=\frac{\left|V_{\Lambda_{i}}\right| \bar{c}_{\Lambda_{i}}}{v_{i}}
$$

Where $v_{i}$ is the local kinematic viscosity and $\bar{c}_{\Lambda_{i}}$ is the swept section chord length. The derivative of Reynolds number with respect to $\Gamma_{j}$ is then 


$$
\frac{\partial R e_{i}}{\partial \Gamma_{j}}=\frac{\bar{c}_{\Lambda_{i}} V_{\Lambda_{i}} \cdot P_{\mathrm{eff}} v_{j i}}{v_{i}\left|V_{\Lambda_{i}}\right|}
$$

The swept section Mach number at any given control point is equal to

$$
M_{i}=\frac{\left|\boldsymbol{V}_{\Lambda_{i}}\right|}{a_{i}}
$$

Where $a_{i}$ is the speed of sound at the control point. The derivative of Mach number with respect to $\Gamma_{j}$ is then

$$
\frac{\partial M_{i}}{\partial \Gamma_{j}}=\frac{\boldsymbol{V}_{\Lambda_{i}} \cdot P_{\mathrm{eff}} \boldsymbol{v}_{j i}}{a_{i}\left|\boldsymbol{V}_{\Lambda_{i}}\right|}
$$

The second half of Eq. 55] is evaluated to yield:

$$
\widetilde{C}_{L_{\Lambda} i} \frac{\partial}{\partial \Gamma_{j}}\left(\left|\boldsymbol{V}_{\Lambda_{i}}\right|^{2}\right)=2 \widetilde{C}_{L_{\Lambda} i} \boldsymbol{V}_{\Lambda_{i}} \cdot P_{\mathrm{eff}} v_{j i}
$$

Simplifying, we obtain the final solution for each element of $[\boldsymbol{J}]$ :

$$
\begin{aligned}
{[\boldsymbol{J}]_{i j}=} & \delta_{i j} 2\left|\boldsymbol{w}_{\boldsymbol{i}}\right|+2 \Gamma_{i} \frac{\boldsymbol{w}_{\boldsymbol{i}} \cdot\left(P_{\mathrm{eff}} \boldsymbol{v}_{\boldsymbol{j} \boldsymbol{i}} \times \boldsymbol{d} \boldsymbol{l}_{\boldsymbol{i}}\right)}{\left|\boldsymbol{w}_{\boldsymbol{i}}\right|}-2 d S_{i} \widetilde{C}_{L_{\Lambda}} v_{i j i} \\
& -\left|\boldsymbol{V}_{\Lambda_{i}}\right|^{2} d S_{i}\left[\widetilde{C}_{L_{\Lambda}, \alpha_{i}} \frac{v_{a i}\left(P_{\mathrm{eff}} \boldsymbol{v}_{\boldsymbol{j} \boldsymbol{i}} \cdot \boldsymbol{u}_{\boldsymbol{n} \boldsymbol{i}}\right)-v_{n i}\left(P_{\mathrm{eff}} \boldsymbol{v}_{\boldsymbol{j} \boldsymbol{i}} \cdot \boldsymbol{u}_{\boldsymbol{a} \boldsymbol{i}}\right)}{v_{a i}^{2}+v_{n i}^{2}}+\widetilde{C}_{L_{\Lambda}, R e_{i}} \frac{\bar{c}_{\Lambda_{i}} v_{i j i}}{v_{i}\left|\boldsymbol{V}_{\Lambda_{i}}\right|}+\widetilde{C}_{L_{\Lambda}, M_{i}} \frac{v_{i j i}}{a_{i}\left|\boldsymbol{V}_{\Lambda_{i}}\right|}\right]
\end{aligned}
$$

where

$$
\begin{gathered}
\boldsymbol{w}_{\boldsymbol{i}} \equiv \boldsymbol{V}_{\Lambda_{i}} \times \boldsymbol{d} \boldsymbol{l}_{\boldsymbol{i}} \\
v_{n i} \equiv \boldsymbol{V}_{\Lambda_{i}} \cdot \boldsymbol{u}_{\boldsymbol{n} \boldsymbol{i}} \\
v_{a i} \equiv \boldsymbol{V}_{\Lambda_{i}} \cdot \boldsymbol{u}_{\boldsymbol{a} i} \\
v_{i j i} \equiv \boldsymbol{V}_{\Lambda_{i}} \cdot P_{\mathrm{eff}} \boldsymbol{v}_{\boldsymbol{j} \boldsymbol{i}}
\end{gathered}
$$

Using this formulation for $J$, we can solve Eq. (50) for $\Delta \boldsymbol{\Gamma}$. We then update $\boldsymbol{\Gamma}$ using

$$
\boldsymbol{\Gamma}_{k+1}=\boldsymbol{\Gamma}_{k}+\psi \Delta \boldsymbol{\Gamma}
$$

where $\psi$ is a relaxation factor and $k$ is the iteration index. A relaxation factor of unity corresponds to accepting the full update. A relaxation factor of less than unity is underrelaxed and tends to more gradual convergence, which may be beneficial for some poorly-behaved situations. We continue iterating until the norm of $\boldsymbol{R}$ falls below some specified threshold. Experience has shown for simple wings, this solution method typically converges to a norm of $\boldsymbol{R}$ of less than $10^{-10}$ within 10 iterations.

Within this nonlinear solution method, no assumption has been made as to the exact model for $\widetilde{C}_{L_{\Lambda}}$. Therefore, the swept lift coefficient can be determined using a simple linear model, an interpolated database of experimental data, or any other method most useful for the designer. All that is required is that derivatives of the swept section lift coefficient can be obtained with respect to angle of attack, Reynolds number, and Mach number at a given condition. 


\section{Calculation of Aerodynamic Forces and Moments}

Once the strength of each horseshoe vortex is known, the forces and moments acting on each wing can be determined. We calculate the inviscid force acting on each bound vortex segment using the vortex lifting law

$$
d \boldsymbol{F}_{\Gamma_{i}}=\rho \Gamma_{i} \boldsymbol{V}_{\Lambda_{i}} \times \boldsymbol{d} \boldsymbol{l}_{i}
$$

The differential moment due to vortex lift is then given by

$$
d \boldsymbol{M}_{\Gamma_{i}}=\boldsymbol{r}_{i} \times d \boldsymbol{F}_{\Gamma_{i}}
$$

where $\boldsymbol{r}_{i}$ is the location of the control point relative to the aircraft center of gravity. To evaluate the total inviscid moment acting on the aircraft, we must also consider the moment due to section aerodynamic properties. The differential moment vector due to section aerodynamic properties is given by

$$
d \boldsymbol{M}_{\text {section }_{i}}=q_{\Lambda_{i}} \bar{c}_{\Lambda_{i}} d S_{i} \widetilde{C}_{m_{\Lambda} i}(\boldsymbol{a}) \boldsymbol{u}_{S_{\Lambda} i}
$$

where

$$
q_{\Lambda_{i}}=\frac{1}{2} \rho_{i}\left|\boldsymbol{V}_{\Lambda_{i}}\right|^{2}
$$

As a first-order approximation, we also predict the viscous drag on an aircraft from a knowledge of the parasitic drag generated by the airfoil sections at a given flow condition. The differential viscous force vector acting on a section is given by

$$
d \boldsymbol{F}_{\mathrm{visc}_{i}}=q_{i} d S_{i} \widetilde{C}_{D_{i}}(\boldsymbol{a}) \boldsymbol{u}_{i}
$$

where

$$
\begin{gathered}
q_{i}=\frac{1}{2} \rho_{i}\left|\boldsymbol{V}_{i}\right|^{2} \\
\boldsymbol{u}_{i}=\frac{\boldsymbol{V}_{i}}{\left|\boldsymbol{V}_{i}\right|}
\end{gathered}
$$

This follows from our previous discussion on the section parasitic drag coefficient for swept wings. The corresponding differential viscous moment vector is then given by

$$
d \boldsymbol{M}_{\mathrm{visc}_{i}}=\boldsymbol{r}_{i} \times d \boldsymbol{F}_{\mathrm{visc}_{i}}
$$

Summing these differential elements across each wing gives the total inviscid and viscous force and moment vectors acting on the aircraft.

\section{Transformation of Forces and Moments to the Wind and Stability Frames}

As the angle of attack and sideslip angle encode successive rotations from the wind frame to the stability frame to the body frame, these angles can be used to determine the vector components of $\boldsymbol{F}$ and $\boldsymbol{M}$ in the wind and stability frames. In the previous section, we obtained $\boldsymbol{F}$ and $\boldsymbol{M}$ in the body-fixed frame. This was due to the ease of defining the geometry of the aircraft in the body-fixed frame. Transforming the forces and moments to the wind and stability frames is a straightforward matter. This transformation can be accomplished using one of two methods: a rotation matrix or a quaternion transformation. For this derivation, we use the definitions of the wind and stability frames given in [15].

\section{Rotation Matrix}

A rotation matrix can be constructed from the directions of the coordinate axes for the wind and stability frames expressed in the body frame. As the body and stability frames differ by a rotation about the body $y$-axis (the angle of this rotation is $\alpha$, the angle of attack), the $y$-axis is the same in the body and stability frames. Similarly, the stability and wind frames differ by a rotation about the stability $z$-axis (the angle of this rotation is $\beta$, the sideslip angle) and so the $z$-axis remains the same in the stability and wind frames [15]. From a knowledge of the body-fixed coordinate axes, $\boldsymbol{i}_{x_{b}}$, $\boldsymbol{i}_{y_{b}}$, and $\boldsymbol{i}_{z_{b}}$, and the freestream direction vector, $\boldsymbol{u}_{\infty}$, we can construct all other coordinate axes. 
The direction of positive lift is opposite the stability and wind z-axes. Lift is defined as being perpendicular to the freestream. However, to allow for a definition of sideforce as well, we say that lift is also perpendicular to the body $y$-axis. Thus

$$
\boldsymbol{u}_{L}=\frac{\boldsymbol{u}_{\infty} \times \boldsymbol{i}_{y_{b}}}{\left|\boldsymbol{u}_{\infty} \times \boldsymbol{i}_{y_{b}}\right|}
$$

The order of the cross product is important for preserving the correct direction of lift. Normalization is required as $\boldsymbol{u}_{\infty}$ and $\boldsymbol{i}_{y_{b}}$ are not guaranteed to be orthogonal. To complete the orthogonal stability coordinate system, we define

$$
\boldsymbol{i}_{x_{s}}=\boldsymbol{u}_{L} \times \boldsymbol{i}_{y_{b}}
$$

Normalization is not needed because $\boldsymbol{u}_{L}$ is guaranteed to be orthogonal to $\boldsymbol{i}_{y_{b}}$ from Eq. (77). The rotation matrix that will transform a vector from the body frame to the stability frame is then

$$
R_{\text {stab }}=\left[\begin{array}{c}
\boldsymbol{i}_{x_{s}}^{T} \\
\boldsymbol{i}_{y_{b}}^{T} \\
-\boldsymbol{u}_{L}^{T}
\end{array}\right]
$$

To obtain the rotation matrix to the wind frame, all that remains is to define the direction of the sideforce, as the wind $x$-axis is opposite $\boldsymbol{u}_{\infty}$. To do this, we simply complete the orthogonal coordinate system formed by $\boldsymbol{u}_{L}$ and $\boldsymbol{u}_{\infty}$.

$$
\boldsymbol{i}_{y_{w}}=\boldsymbol{u}_{L} \times \boldsymbol{u}_{\infty}
$$

Again, normalization is not needed. The rotation matrix that will transform a vector from the body frame to the wind frame is then

$$
R_{\text {wind }}=\left[\begin{array}{c}
-\boldsymbol{u}_{\infty}^{T} \\
\boldsymbol{i}_{y_{w}}^{T} \\
-\boldsymbol{u}_{L}^{T}
\end{array}\right]
$$

The lift and drag forces will then be the negatives of the wind $x$ - and $z$-forces, respectively, as implied by the negative signs on $\boldsymbol{u}_{L}$ and $\boldsymbol{u}_{\infty}$ in Eq. (81).

A vector, $v_{b}$ expressed in the body frame can then be transformed to the wind or stability frames simply by premultiplying by the appropriate rotation matrix.

$$
\begin{gathered}
\boldsymbol{v}_{s}=R_{\text {stab }} v_{b} \\
v_{w}=R_{\text {wind }} v_{b}
\end{gathered}
$$

\section{Quaternion Transformation}

As $\alpha$ and $\beta$ describe two successive rotations, the total rotation can be encoded by a quaternion. Phillips [16] gives formulas for calculating the orientation quaternion of an aircraft from its Euler angles, which we can adapt to frame transformations based on $\alpha$ and $\beta$. To go from the wind frame to the stability frame, there is a rotation about the $z$-axis by $\beta$. This is analogous to the heading angle when transforming from the Earth-fixed frame to the body frame. To go from the stability frame to the body frame there is a rotation about the $y$-axis by $\alpha$, which is analogous to the elevation angle. The bank angle has no analogue in this case. Using Phillips' equations, the quaternion which encodes a rotation from the wind frame to the body frame is given by

$$
\boldsymbol{q}_{\text {wind }}=\left[\begin{array}{c}
C_{\alpha / 2} C_{\beta / 2} \\
-S_{\alpha / 2} S_{\beta / 2} \\
S_{\alpha / 2} C_{\beta / 2} \\
C_{\alpha / 2} S_{\beta / 2}
\end{array}\right]
$$


where $C$ and $S$ denote the cosine and sine, respectively, of the subscripted quantity. Note we use the scalar-first definition for the quaternion in the current work. The quaternion encoding a rotation from the stability frame to the body frame will then be the same except with $\beta$ set to zero.

$$
\boldsymbol{q}_{\mathrm{stab}}=\left[\begin{array}{c}
C_{\alpha / 2} \\
0 \\
S_{\alpha / 2} \\
0
\end{array}\right]
$$

To transform a vector, $\boldsymbol{v}_{b}$, from the body frame to either the wind or stability frame, it is simply necessary to perform an inverse quaternion transform using the appropriate quaternion [16].

$$
\begin{gathered}
\boldsymbol{v}_{w}=\boldsymbol{q}_{\text {wind }} \otimes\left(\boldsymbol{v}_{b} \otimes \boldsymbol{q}_{\text {wind }}^{*}\right) \\
\boldsymbol{v}_{s}=\boldsymbol{q}_{\mathrm{stab}} \otimes\left(\boldsymbol{v}_{b} \otimes \boldsymbol{q}_{\mathrm{stab}}^{*}\right)
\end{gathered}
$$

where * denotes the quaternion conjugate. As this method requires evaluation of trigonometric functions, the rotation matrix approach is preferred for computational speed.

\section{Lifting-Line Placement}

There is some ambiguity as to where the lifting-line (i.e. the locus formed by the bound vortex segments) is to be placed relative to the actual geometry of the wing. In Prandtl's original theory, the lifting-line is placed at the quarter-chord of the wing [1]. However, since Prandtl's theory encompasses only straight wings, the location of the lifting-line relative to the actual wing has no impact on the solutions obtained when considering a single wing.

Others have put forward suggestions for where to place the lifting-line. Phillips and Snyder [4], and Reid and Hunsaker [5] both propose that the lifting-line should be coincident with the LAC of the wing but offer no justification for doing so. For swept wings, Reid and Hunsaker [5] use Küchemann's correction to offset the LAC from the LQC.

Weissinger [17] places the lifting-line on the quarter-chord line and enforces a flow-tangency condition at the three-quarter-chord. Within his work, Weissinger cites Helmbold in a then yet unpublished work saying that for an infinitely wide plate, "the downwash of the lifting surface and the downwash of the lifting line located at the one-quarter chord position are equal to each other at the three-quarter chord distance" [17]. Thus in this case, the placement of the lifting-line on the quarter-chord stems from analytical results of potential flow theory. The approach of placing the lifting-line on the quarter-chord is also followed by Rosen and Rand [10], albeit on an infinite, straight-swept wing.

Guermond [6], in developing an analytic lifting-line theory for swept wings, places the lifting-line such that it represents the "spanwise mean geometry" (i.e. it is placed on the half-chord line). In presenting a numerical application of Guermond's method, Devinant and Gallois [18] state that, within Guermond's method, the lifting line may be placed anywhere along the chord. They choose to place it on the quarter-chord in order to facilitate direct comparison with Prandtl's classical theory. In practice, Devinant and Gallois model the lifting-line of a straight swept wing as lying on the quarter-chord except at the root. At the root, they join the two halves of the lifting-line using a small cosine curve, so as to ensure a continuous radius of curvature along the lifting-line [18].

Using the G-H method, the placement of the lifting-line does have a noticeable impact on the predicted lift distribution. Figure 5 shows the variation in the predicted lift distribution for a straight swept wing when the lifting-line is placed on the LQC compared to when the lifting-line is placed on the LAC (determined by Küchemann's correction [19]). These are both plotted against experimental data from Weber et al. [7]. As can be seen, the predicted lift distribution has an increase in lift at the root when the lifting-line is placed on the LAC. This is clearly not present when the LQC is used and in the experimental data. Other experimental and high-order data (see, for example [5, 18, 20]) consistently show a characteristic dip in lift at the root of a swept wing, rather than an increase.

We hypothesize that the increase in the lift distribution seen at the root when the lifting-line is placed on the LAC is due to the lifting-line becoming more nearly perpendicular to the flow at the root. This would tend to increase the predicted lift in three ways. First, the bound vortex segment becoming more perpendicular to the freestream increases the lift predicted by the vortex lifting law for a given vortex strength. Second, this also leads to an increase in the swept section angle of attack near the root. And third, the effective freestream velocity seen by sections near the root is greater. 


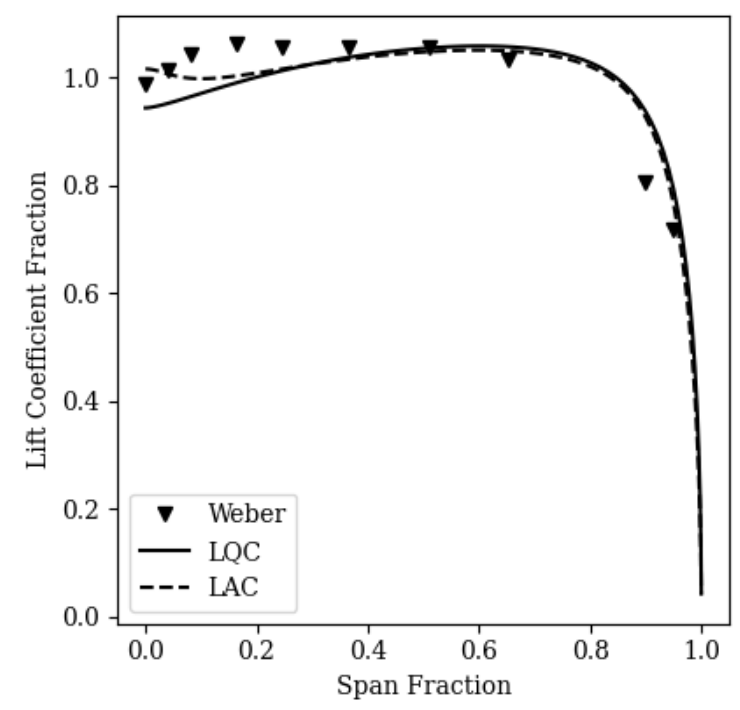

Fig. 5 Lift distributions on a swept wing showing the effect of the placement of the lifting-line on the final result. Experimental data from Weber et al. are shown for comparison [7].

We therefore propose that, since the lifting-line algorithm condenses the full geometry of the wing onto a single line (the lifting-line), changing the position of the lifting-line away from a constant chord-wise station amounts to changing the geometry of the wing. For example, placing the lifting-line on the LAC as defined by Küchemann's correction would be equivalent to modeling a wing which has a rounded out root section and tips with decreased sweep. Therefore, we suggest it is most appropriate to place the lifting-line at a constant chord-wise station along the wing, thereby preserving the geometry of the wing.

We recommend placing the lifting-line on the wing LQC. This is standard practice for lifting-line methods and already represents a first-order approximation of the wing LAC. This placement also facilitates straightforward calculation of moment contributions due to sectional properties. As the moment coefficient of an airfoil section is most often reported about the quarter-chord, no shifting of this moment is needed if the control points are placed at the quarter-chord. This convention is followed throughout the rest of this work. However, further research into these hypotheses is desired.

\section{Validation}

The G-H method has been implemented in a user-friendly Python package called MachUpX [21]. We use MachUpX to obtain all results from the G-H method presented in this work. To the authors' knowledge, MachUpX has already also been used for aeroelastic analysis on the NASA common research model [22], design and simulation of a small unmanned aerial vehicle [15], twist optimization for a specified lift distribution on swept and unswept wings [23], and design and analysis of a morphing wing aircraft [24].

As recommended by Reid and Hunsaker [5], we use a non-dimensional joint length of 0.15 and a blending distance of 0.25 in all cases.

\section{A. Grid Convergence}

In expanding the R-H method into the G-H method, we have ensured that the effective lifting-line still has zero curvature in the neighborhood of the control point and that the jointed vortices remain perpendicular to the effective lifting-line. Thus, the G-H method is still grid convergent. To demonstrate this, we present a brief example.

Fig. 6 shows the circulation (vortex strength) distribution as predicted by MachUpX at various grid sizes for a wing with $45^{\circ}$ sweep and $5^{\circ}$ dihedral. This wing had an aspect ratio of 8 and a constant NACA 0010 section (viscous lift slope of 6.4336). This wing also had a mounting angle of $5^{\circ}$ with $5^{\circ}$ of linear twist starting at the mid-span. The case was run at an angle of attack of $5^{\circ}$ and a freestream velocity of $100 \mathrm{ft} \mathrm{s}^{-1}$. Fig. 6a shows the circulation predicted using the P-S 
method and Fig. 6b shows the circulation predicted using the G-H method. The P-S method fails to grid converge and attempts to force the circulation to zero at the wing root. On the other hand, the results given by the G-H method grid converge, showing the conditions imposed by Reid and Hunsaker [5] are still satisfied.

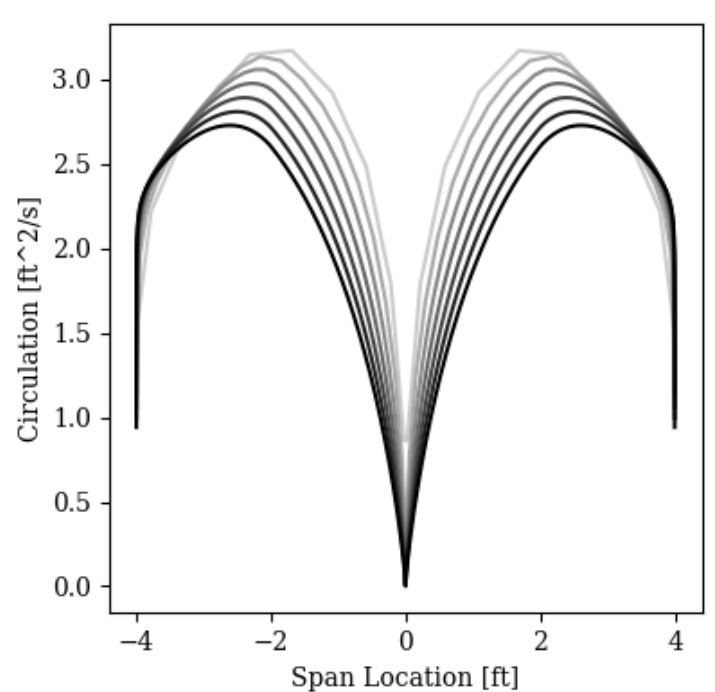

(a)

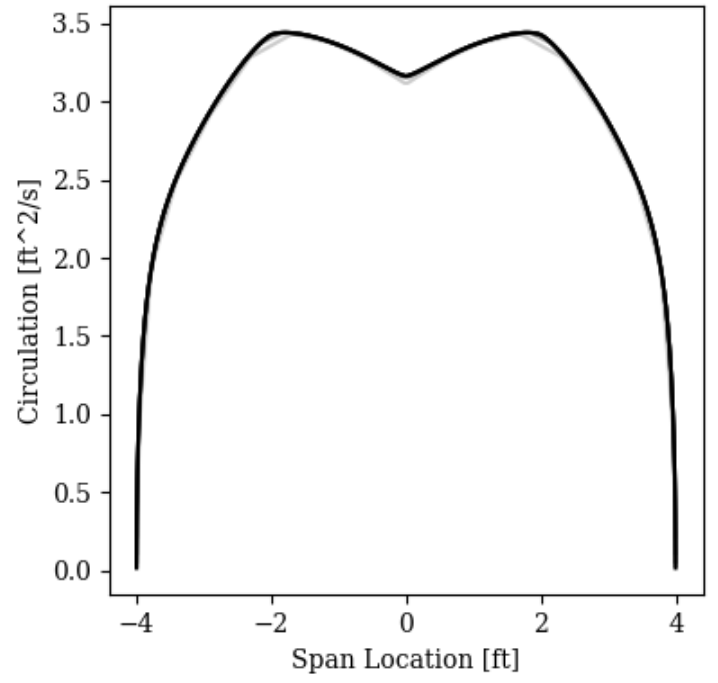

(b)

Fig. 6 Circulation distribution for a swept wing with dihedral and twist using grid sizes varying from 10 (light grey) to 640 (black) nodes per semispan using the (a) P-S and (b) G-H methods.

Based on this and other convergence studies, we use a grid resolution of 80 cosine-clustered points per semispan in all subsequent cases.

\section{B. Literature Comparisons}

In this section, we compare results obtained from the $\mathrm{G}-\mathrm{H}$ method to results available in the literature. These results encompass predictions of overall aerodynamic coefficients, as well as coefficient distributions, for forward-swept, unswept, and backward-swept wings. These comparisons provide a good reference for evaluating the accuracy and usefulness of the G-H method.

\section{Case 1: Weber and Brebner}

Weber and Brebner investigated the effect of changing root section properties (i.e. thickness, camber, and twist) on the overall lift distribution of a swept-back wing [7]. They report the lift coefficient distribution due to pressure for three wings, as well as the total lift and drag coefficients for each at varying angles of attack. Each wing had the same planform with a constant 45 degree sweep, a constant 20 inch chord, and a 98 inch span. Weber and Brebner give limited data for all but the first wing, wing $\mathrm{A}$, and so we provide comparisons for this wing only.

Wing A investigated by Weber and Brebner. had a constant RAE101 airfoil section. For this comparison, we use a viscous section lift slope of 5.935 for the RAE101, as reported by Reid and Hunsaker [5]. Since Weber and Brebner. report only values of pressure drag, we specify the wing section in MachUpX as having no skin friction drag. Figure 7 shows the section lift coefficient (normalized by the total lift coefficient) as predicted by the R-H method [5], PAN AIR [25] (obtained by Reid and Hunsaker [5]), and the G-H method, compared to the experimental data from Weber and Brebner for wing A at an angle of attack of $4.2^{\circ}$. There is very close agreement between all four sets of data. The lifting-line methods tend to predict the lift distribution as being higher towards the tip and lower towards the root than the experimental data. Similar behavior is seen in the results from PAN AIR, though the shift is less pronounced. This is likely due to the development of significant spanwise flow towards the wingtips, which may affect the true lift distribution and which lifting-line theory is unable to model. This hypothesis is supported by observing that PAN AIR does predict a lower lift coefficient at the tip; since PAN AIR is a panel solver, it can capture more spanwise effects [5]. 


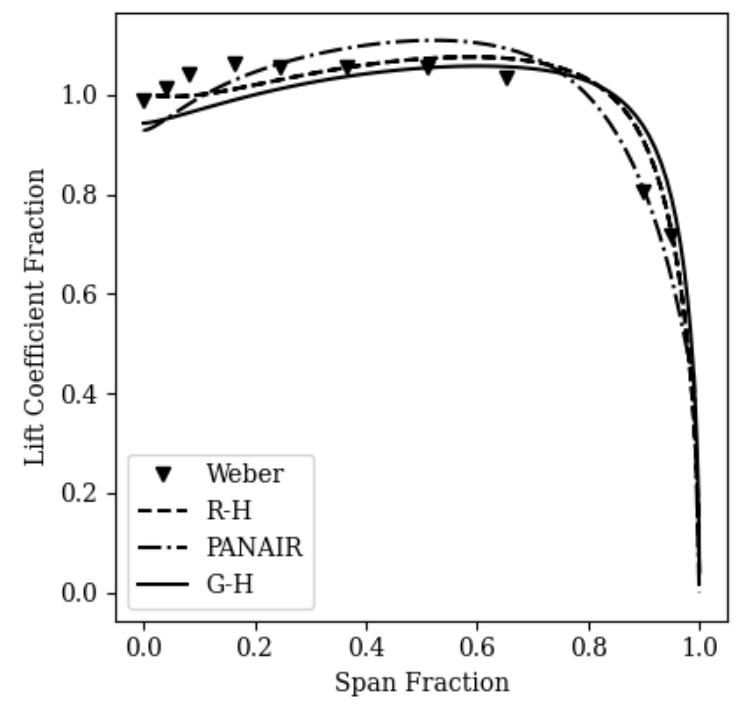

Fig. 7 Lift coefficient fraction as a function of span for Weber's wing A as predicted by the R-H and G-H methods, and PAN AIR, compared to experimental data.

Figure 8 shows the lift distribution for wing A as a function of span as predicted by the G-H method at varying angles of attack. These are plotted against experimental results from Weber et al. The lift distribution remains quite accurate on the inner half of the wing, even up to high angles of attack. However, as the angle of attack is increased, the G-H method overpredicts the section lift on the outer half.

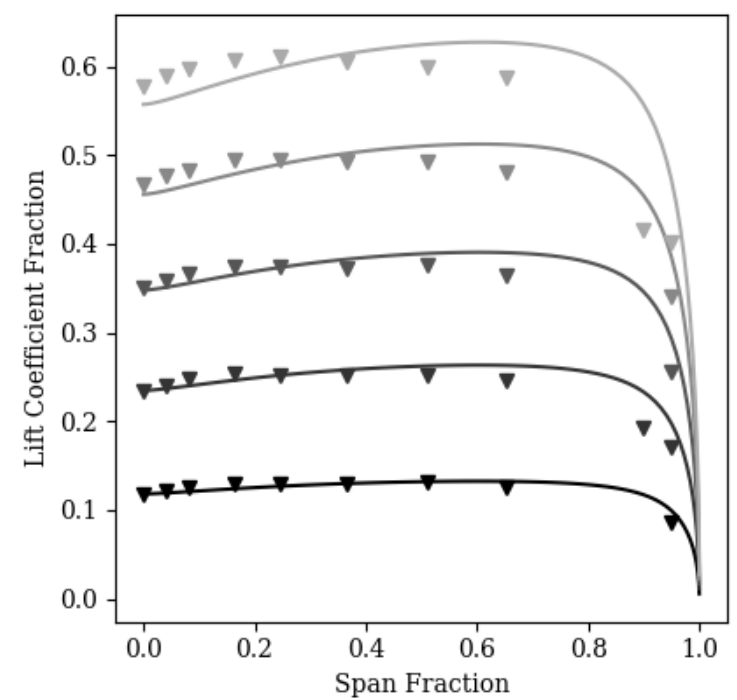

Fig. 8 Section lift coefficient as a function of span for Weber and Brebner's wing A as predicted by the G-H method at angles of attack ranging from 2.1 (black) to 10.5 (grey) degrees. The solid lines are the distribution predicted by the G-H method and the triangles are the experimental results.

As shown in Fig. 9a, the G-H method overpredicts the total lift at higher angles of attack. This is also seen in Fig. 8 Since within the G-H method we assume a value for the linear lift slope of the wing section, the discrepancies seen may 
be due to incorrect prediction of the section lift slope. However, the discrepancy could also simply be due to increased spanwise effects at high angles of attack, as discussed previously.

Figure $9 \mathrm{~b}$ shows the pressure drag as a function of lift coefficient for wing A. Since Weber and Brebner [7] report only pressure drag, we would expect the drag predicted by the G-H method to closely match the experimental results. However, there is a large discrepancy here. This is likely due to viscous phenomena having a non-negligible effect on the pressure data taken.

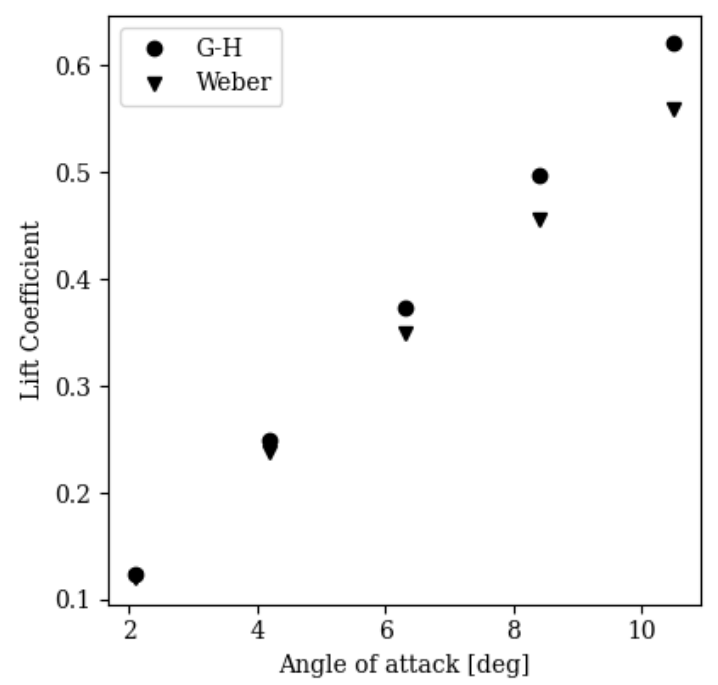

(a)

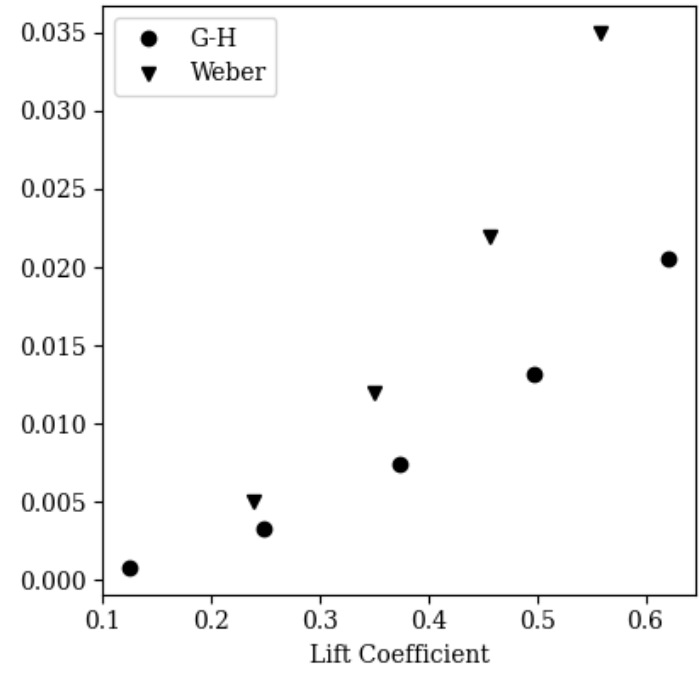

(b)

Fig. 9 Lift coefficient versus angle of attack (a) and drag polar (b) for wing A as predicted by the G-H method compared to experimental data.

\section{Case 2: Van Dorn and DeYoung}

Van Dorn and DeYoung [20] collected lift distribution data on forward and backward swept wings in order to test the accuracy of various numerical methods for predicting the aerodynamics of swept wings. In the current work, we compare only to the experimental results they report.

The geometry of these wings is reported by McCormack and Stevens [11]. These wings were constructed using available wing panels with custom manufactured center and tip sections to achieve the desired sweep. The main wing panels had a section which varied from a NACA 0015 at the root to a NACA 23009 at the tip. The root and tip sections were manufactured such that the aspect ratio was the same between each of the 5 wings [11]. Table 1 gives the dimensions of these 5 wings.

Table 1 Relevant dimensions for the 5 wings investigated by Van Dorn and DeYoung [11].

\begin{tabular}{lcccc}
\hline Wing & Sweep, $^{\circ}$ & Root Chord, ft & Tip Chord, ft & Span, ft \\
\hline 1 & -45 & 15.40 & 5.79 & 32.38 \\
2 & -30 & 11.34 & 4.59 & 36.39 \\
3 & 0 & 8.71 & 4.73 & 30.53 \\
4 & 30 & 10.53 & 4.65 & 36.06 \\
5 & 45 & 13.34 & 5.59 & 33.56 \\
\hline
\end{tabular}

Van Dorn and DeYoung report a constant section lift slope of 5.9015 along the span [20], which we use here. In comparing to numerical methods, Van Dorn and DeYoung remove all effects of what they call the base loading, which is 
the lift distribution on the wing at zero degrees angle of attack. This was done in order to be able to model the wings in the various numerical methods as flat plates (i.e. no geometric or aerodynamic twist). The experimental data is corrected accordingly [20]. As such within MachUpX, we use a single airfoil section along the span of the wing with a zero-lift angle of attack of zero. We specify the airfoil sections with no drag terms, since Van Dorn and DeYoung report only lift due to pressure [20].

Within MachUpX, we turn off corrections to the section coefficients due to sweep. This is because the wings investigated by Van Dorn and DeYoung were swept via a solid-body rotation, and so the section geometry normal to the LQC remains the same [11]. McCormack and Stevens also report that no attempt was made to smooth the wing panels used in their investigation, and so various sources of roughness, such as access panels and panel edges, were present on their test models [11]. We make not attempt to account for this roughness and so expect the results from MachUpX to underpredict viscous contributions for these wings.

As seen in Fig. 10, the G-H method predicts the spanwise lift distribution on an unswept wing remarkably well in comparison to experimental data. This is expected as lifting-line theory is best suited for unswept wings. The discrepancies seen in Fig. 10 between the numeric and experimental data are likely within the error of the experimental data.

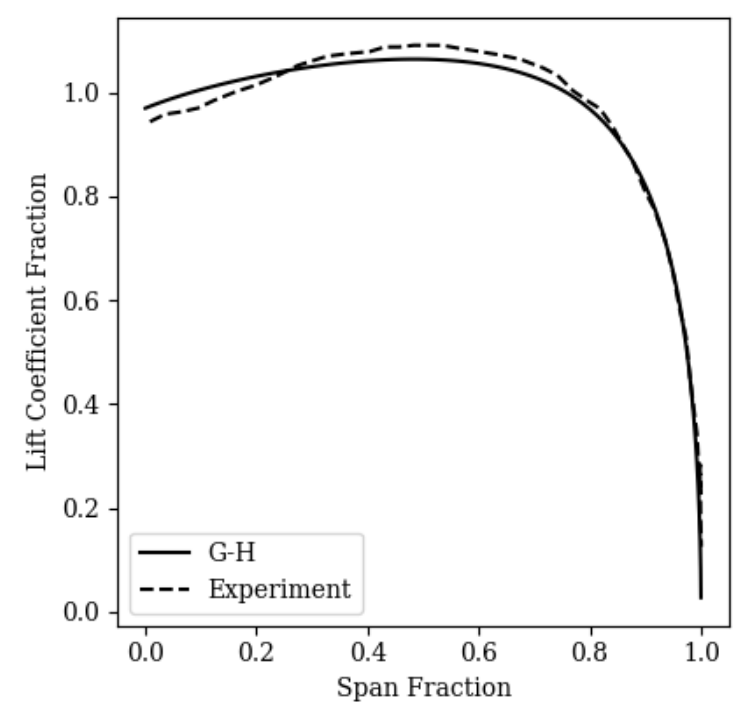

Fig. 10 Lift coefficient fraction as a function of span as predicted by the G-H method as a function of span for a wing with no sweep

Figures $11 \mathrm{a}$ and $11 \mathrm{~b}$ show the predicted and experimental lift distributions for the backward swept wings investigated by Van Dorn and DeYoung. For these wings, the G-H method underpredicts the lift near the root and overpredicts the lift near the tip of the wing. This is an interesting contrast to the numerical results reported by Van Dorn and DeYoung. As they point out, the numerical methods they investigated (the methods of Faulkner, Mutterperl, and Weissinger) consistently overpredict the lift at the root and underpredict the lift at the tip of the backward swept wings [20].

Figures $12 \mathrm{a}$ and $12 \mathrm{~b}$ show the predicted and experimental lift distributions for the forward swept wings investigated by Van Dorn and DeYoung. For these wings, the G-H method overpredicts the lift at the root and underpredicts the lift at the tip. This follows the trend reported by Van Dron and DeYoung discussed previously [20].

\section{General Remarks on Comparisons}

Experimental research [26, 27] has shown that the vortical flowfield structure is much more complicated for swept wings than for unswept wings. In addition to the wingtip vortices which dominate the wake of an unswept wing and induce downwash, swept wings also generate leading and trailing edge vortices. Leading edge vortices form over the leading edge of swept wings at an angle of attack [26]. These complex vortical structures are not modeled in lifting-line theory and so may be a source of error in predicting the lift distribution on swept wings. Future research into 


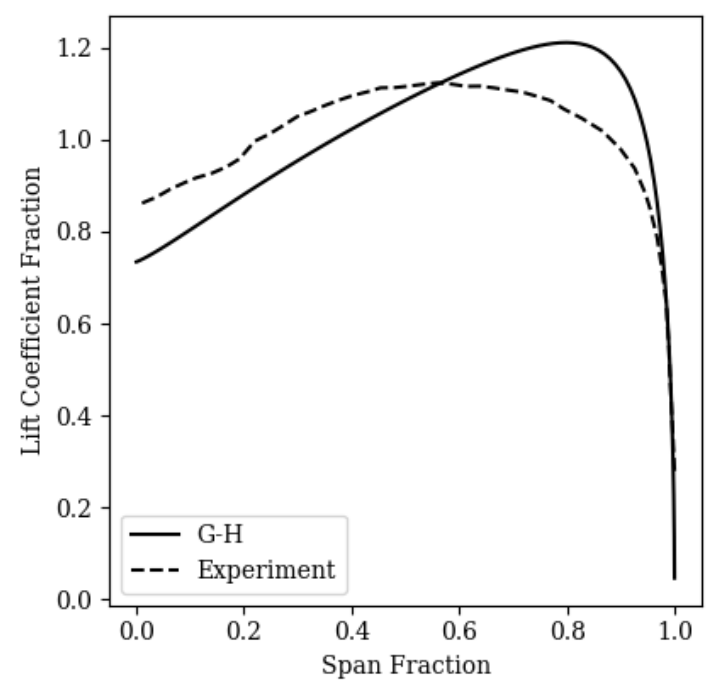

(a)

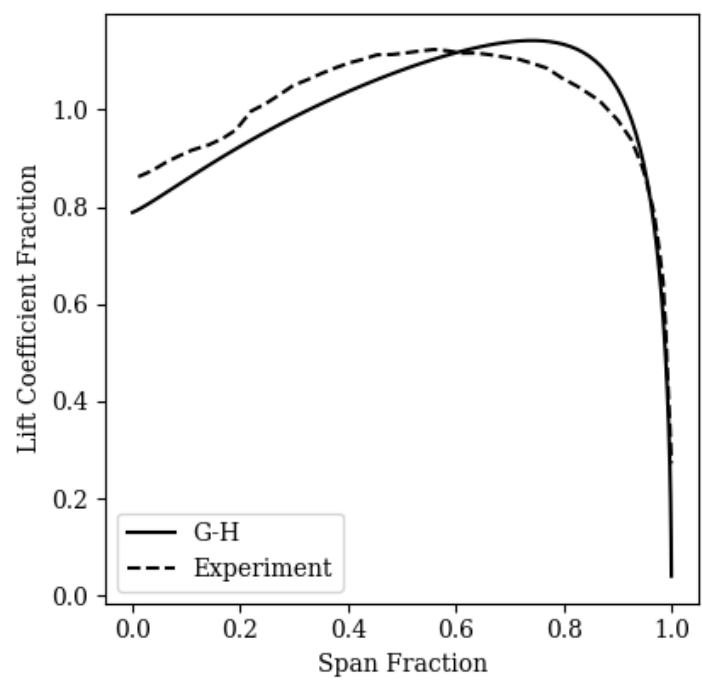

(b)

Fig. 11 Lift coefficient fraction as a function of span as predicted by the G-H method compared to experimental data for two backward swept wings with (a) 45 and (b) 30 degrees of sweep.

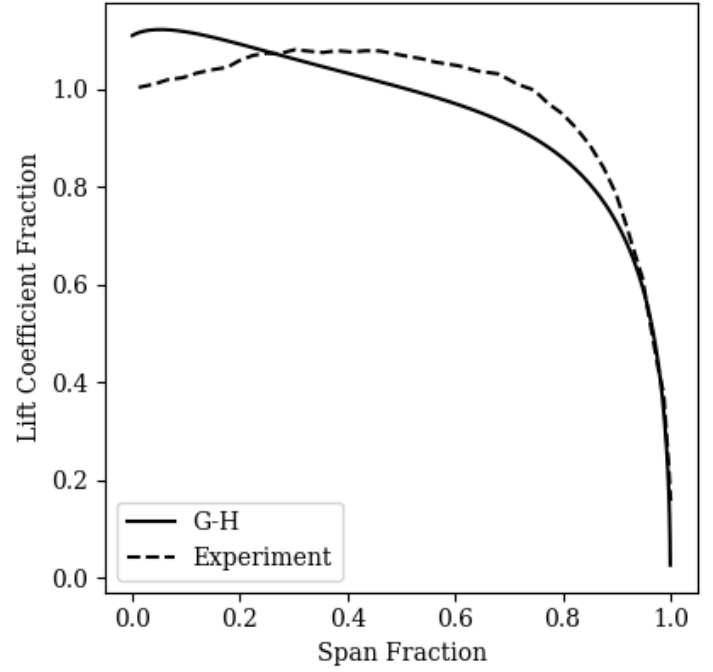

(a)

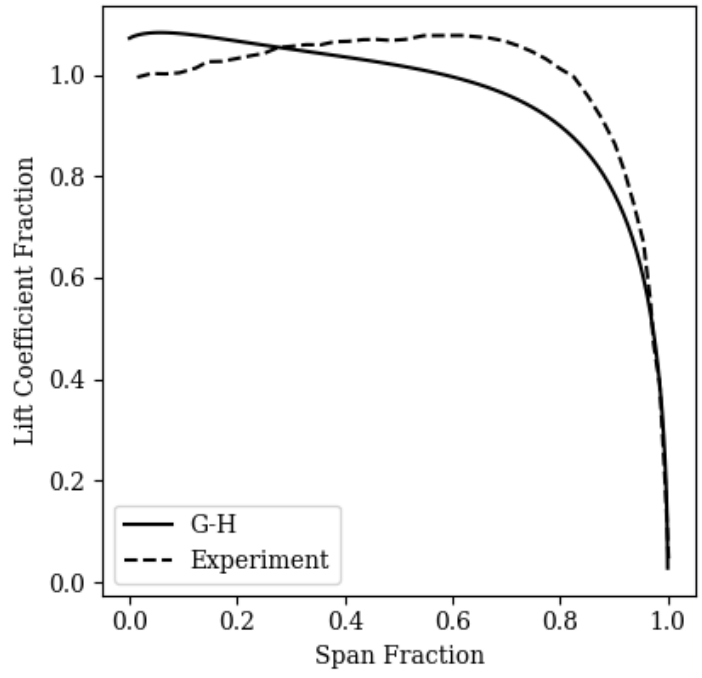

(b)

Fig. 12 Lift coefficient fraction as a function of span as predicted by the G-H method compared to experimental data for two forward swept wings with (a) 45 and (b) 30 degrees of sweep.

incorporating leading and trailing edge vortex effects into a general numerical lifting-line method for swept wings could lead to great improvements in its accuracy.

A viscous vortex phenomenon called bursting may also be responsible for some of the discrepancies observed for forward swept wings. At a certain point along the axis of a vortex, the vortex transitions to lower-energy, turbulent flow [27]; this event is referred to as bursting. This bursting has been connected with a change in the pressure distribution on the surface of the wing [28]. As bursting is a viscous phenomenon, its effect is not modeled within lifting-line methods 
and so may also be a source of error when comparing to experimental results.

\section{Conclusion}

The Goates-Hunsaker method for solving the general lifting-line problem can be successfully applied as presented in this work to an arbitrary number of wings with arbitrary sweep, dihedral, and twist distributions. The G-H method also incorporates corrections to swept section coefficients based on thin-airfoil theory, which may be applied to the airfoil sections regardless of local sweep. In this work, we have shown how both a linearized approximation to the lifting-line equation and a nonlinear improvement method may be used within the G-H method to solve for the vortex strength distribution of a given set of wings. Using these solution methods, we have shown how the G-H method is sensitive to the chord-wise placement of the lifting-line and recommend it be placed on the LQC. Compared to published data, the G-H method provides accurate predictions of the lift distribution for wings with various sweep angles.

\section{Acknowledgments}

The authors would like to thank Jackson Reid for his invaluable input on this research as well as for paving the way in expanding numerical lifting-line methods to wings with sweep. They would also like to thank the members of the USU AeroLab, as well as Francois Fortin (National Research Council of Canada) and Christina Harvey (University of Michigan), for their tireless efforts and patience testing MachUpX. This work was funded by the U.S. Office of Naval Research Sea-Based Aviation program (Grant No. N00014-18-1-2502) with Brian Holm-Hansen as the program officer.

\section{References}

[1] Prandtl, L., "Tragflügel Theorie,” Nachrichten von der Gesellschaft der Wisseschaften zu Göttingen, 1918, pp. $451-477$.

[2] Anderson, J., "Fundamentals of Aerodynamics," McGraw-Hill Education, New York, 2017, Chap. 4.8, 6" ed., pp. 356-365.

[3] Phillips, W. F., “Mechanics of Flight,” John Wiley \& Sons, Hoboken, 2010, Chap. 1.9, $2^{\text {nd }}$ ed., pp. 94-107.

[4] Phillips, W. F., and Snyder, D. O., "Modern Adaptation of Prandtl's Classic Lifting-Line Theory," Journal of Aircraft, Vol. 37 , No. 4, 2000.

[5] Reid, J. T., and Hunsaker, D. F., "A General Approach to Lifting-Line Theory, Applied to Wings with Sweep," AIAA Scitech 2020 Forum, AIAA, Orlando, FL, 2020, p. 28.

[6] Guermond, J.-L., “A Generalized Lifting-Line Theory for Curved and Swept Wings,” Journal of Fluid Mechanics, Vol. 211, 1990, pp. 497-513.

[7] Weber, J., and Brebner, G., “Low-Speed Tests on 45-deg Swept-Back Wings, Part I," Technical Report 2882, Aeronautical Research Council Reports and Memoranda, 1958.

[8] Reid, J. T., A General Approach to Lifting-Line Theory, Applied to Wings with Sweep, Utah State University, 2020. PhD Thesis.

[9] Küchemann, D., and Weber, J., “The Subsonic Flow Past Swept Wings at Zero Lift Without and With Body,” Technical Report 2908, Aeronautical Research Council Reports and Memoranda, 1958.

[10] Rosen, A., and Rand, O., "The Aerodynamic Behavior of Swept Wings: Another Point of View," Journal of Aircraft, Vol. 22, No. 1, 1985, pp. 83-85.

[11] McCormack, G. M., and Stevens, V. I., "An Investigation of the Low-Speed Stability and Control Characteristics of SweptForward and Swept-Back Wings in the Ames 40- by 80-foot Wind Tunnel," NACA Research Memorandum No. A6K15, June 1947.

[12] Phillips, W. F., "Mechanics of Flight," John Wiley \& Sons, Hoboken, 2010, Chap. 1.9, 2 ${ }^{\text {nd }}$ ed., pp. $28-32$.

[13] Gallay, S., and Laurendeau, E., "Preliminary-Design Aerodynamic Model for Complex Configurations Using Lifting-Line Coupling Algorithm," Journal of Aircraft, Vol. 53, No. 4, 2016.

[14] Ghasemi, A., S; Mosahebi, and Laurendeau, E., "A Two-Dimensional/Infinite Swept Wing Navier-Stokes Solver," 52nd Aerospace Sciences Meeting, AIAA, National Harbor, MD, 2014. 
[15] Thurgood, J., and Hunsaker, D. F., "Sensitivity and Observability of Aerodynamic Parameters of a Flying Wing Using Simulation," AIAA Scitech 2021 Forum, AIAA, Virtual, 2021.

[16] Phillips, W. F., “Mechanics of Flight,” John Wiley \& Sons, Hoboken, 2010, Chap. 1.9, 2 ${ }^{\text {nd }}$ ed., pp. $1004-1012$.

[17] Weissinger, J., “The Lift Distribution of Swept-Back Wings,” NACA Technical Memorandum No. 1120 , March 1947.

[18] Devinant, P., and Gallois, T., "Swept and Curved Wings: A Numerical Approach Based on Generalized Lifting-Line Theory," Computational Mechanics, Vol. 29, 2002, pp. 322-331.

[19] Moorthamers, B., and Hunsaker, D. F., “Accuracy of Küchemann's Prediction for the Locus of Aerodynamic Centers on Swept Wings," AIAA Scitech 2020 Forum, AIAA, Orlando, FL, 2020.

[20] Van Dorn, N. H., and DeYoung, J., "A Comparison of Three Theoretical Methods of Calculating Span Load Distribution of Swept Wings," NACA Research Memorandum No. A7C31, June 1947.

[21] AeroLab, "MachUpX: Fast and accurate aerodynamic modelling using lifting-line theory.", 2020. https://www . github. com/usuaero/MachUpX

[22] Taylor, J., and Hunsaker, D. F., "Characterization of the Common Research Model Wing for Low-Fidelity Aerostructural Analysis,” AIAA Scitech 2021 Forum, AIAA, Virtual, 2021.

[23] Fortin, F., and Goates, C., "Extraction of Geometric Twist for Given Spanwise Load Using Low-Order Methods," AIAA Scitech 2021 Forum, AIAA, Virtual, 2021.

[24] Snow, S., and Hunsaker, D. F., "Design and Performance of a 3D-Printed Morphing Aircraft," AIAA Scitech 2021 Forum, AIAA, Virtual, 2021.

[25] Epton, M. A., and Magnus, A. E., "PAN AIR: A computer program for predicting subsonic or supersonic linear potential flows about arbitrary configurations using a higher order panel method. Volume 1: Theory document," NASA-CR-3251, 1981.

[26] Breitsamter, C., and Laschka, B., "Vortical Flowfield Structure at Forward Swept-Wing Configurations," Journal of Aircraft, Vol. 38, 2001, pp. 193-207.

[27] Lambourne, N. C., and Bryer, D. W., "The Bursting of Leading-Edge Vortices-Some Observations and Discussion of the Phenomenon,” Technical Report 3282, Aeronautical Research Council Reports and Memoranda, 1961.

[28] Owen, T. B., "Techniques of pressure-fluctuation measurements employed in the R.A.E. low-speed wind tunnels," AGARD Report 172, 1961. 